\title{
Unsteady Numerical Simulation of an Axial Piston Pump for Lubricating Greases using Dynamic Meshes
}

\author{
Menéndez Blanco, A. ${ }^{(1)}$ \\ Fernández Oro, J.M. ${ }^{(2)}$ \\ ${ }^{(1)}$ Samoa Industrial S.A. \\ Pol. Ind. Porceyo, Camino del Fontán s/n, 33392, Gijón (Asturias), Spain. \\ a.menendez@samoaidustrial.com \\ (2) Universidad de Oviedo, Área de Mecánica de Fluidos. \\ Campus de Viesques, 33271, Gijón (Asturias), Spain. \\ jesusfo@uniovi.es
}

\begin{abstract}
This paper presents the numerical simulation of an axial piston pump for lubricating greases. The model, based on the dynamic mesh technique, describes the unsteady displacement of the piston using a layering algorithm. The pump driven velocity has been modified using a parametric user defined function (UDF) that covers the whole range of operating points for the pump, as a function of the discharged outlet pressure.

The leakage flow across the feeder plate gap has been analyzed in detail, monitoring its evolution in both forward and backward strokes as a function of the pressure in the adjacent chambers. The comparison between the numerical results and the predictions from the theoretical formulations in the literature for non-newtonian fluids in annular ducts has confirmed the accuracy of the simulation and the correct selection of the discretization for small gaps and inner passages. In addition, the analysis of the flow delivered overtime has provided the numerical distribution and mean values expected from the experimental performance curves supplied by the manufacturer.

Finally, the flow conditions in the aspirating region of the pump have been studied in terms of dynamic viscosity and grease fluidity. It has been observed that the fluidity is restricted to those zones presenting large areas of velocity gradients, like the feeder gap and the vicinity of the aspirating inlet, especially close to the solid walls of pump and drum. For the intermediate positions of both advancement and returning stages, when the piston velocity is maximum, the obtained maps reveal that the induced suction reach significant portions of grease within the drum, similar in distance to the pump diameter, and confirms the order of magnitude estimated with an inspectional analysis of the governing equations of grease motion in the aspirating zone.
\end{abstract}

Keywords: numerical simulation, lubricating grease, axial piston pump, CFD, dynamic mesh, layering technique, leakage flow, fluidity, non-newtonian pseudoplastic flow, pump aspiration. 


\section{INTRODUCTION}

For years, greases have been used as lubricating fluids in a number of industrial applications due to their lubricating capacity and lack of fluidity when stayed at rest (null tensor stresses). This makes them particularly suitable to lubricate open cavities without leakage risk. Unfortunately, this lack of fluidity has been always a clear disadvantage for pumping and delivering grease, especially when high pressures are required for a wide range of applications.

The pumps employed for these purposes are robust equipments, designed to handle the extremely high pressures associated to the lubricating operations. The air-operated axial piston generates the volumetric displacement in every stroke, which according to the driven velocity of the reciprocating mechanism, delivers the corresponding flow rate. Typically, these lubricating pumps are composed of two adjacent chambers. The first one is a depressurized single chamber that injects the grease into a second chamber during the forward stroke. When the piston pump is moving back, this second chamber, fully isolated and thus without any leakage flow, is progressively collapsed, venting the flow rate at the required operating pressure (grease acting as an incompressible flow according to Pascal's principle). This design, using two consecutive chambers in a row, avoids the presence of leakage flow during the backward stroke, when the pump is really providing the grease flow rate at high-pressure, but it is susceptible from suffering partial cavitation and breaking-off the fluid continuum during the returning.

Regarding the operation of these pumps, manufactured with an accurate length of the external tube for the different types of commercial drums, they are usually mounted over the tap of the grease drums. The low fluidity of the greases, even more conditioned when operated at low temperatures, requires the employment of follower plates, which are even pushed by strong pneumatic cylinders to convey the grease much more. When these elements are not included, it is very common the arising of important problems in the aspirating region during the piston rising, leading to oscillation or pulsating injection of grease, partial vacuum in the drum or severe compacting of solid packages of grease close to the aspiration.

However, this kind of industrial evidences has a difficult characterization because typical grease is a highly-viscous, opaque fluid, with a variable behaviour according to the working temperature or the velocity gradient. Moreover, the high-pressures involved in delivering so reduced flow rates are also a major inconvenience for obtaining reliable values of flow rate or velocity in the small gaps of the pump. Complementarily, the rough operational ranges used in these pumps, as well as the environmental or industrial conditions associated to their use, have extremely limited the development of particular methodologies for their analysis. Nevertheless, some new recent methodologies have arisen for the computational study of volumetric pumps that can be successfully applied for this kind of lubricating pumps with reciprocating operation principle (Houzeaux and Codina [1], Vande Voorde et al. [2], Riemslagh et al. [3]). Since positive displacement pumps operate generating and destroying small volumes, these methodologies require remeshing operations to implement the generation and collapse of cells as the piston displaces overtime (Strasser [4], Kumar et al. [5], Hyun et al. [6]).

This paper describes the numerical modelling of an axial piston pump by means of a remeshing technique that employs a layering method to implement the reciprocating displacement of the piston pump. According to the pump geometry, it has been considered an axisymmetric model of the inner parts within the pump housing, including the outer zones of the aspirating inlet where 
the grease fluidity will be analysed as a function of the operational parameters. In this case, the grease will be modelled as a non-newtonian, pseudoplastic fluid, with both consistency and behaviour indexes known, following the Otswald-de Waele expression. In order to validate the model, the numerical results will be compared with the performance curves of the pump, obtained experimentally, paying particular attention to the pump performance when working at free delivery.

In addition, the leakage flow through the gap of the feeder during back-and-forth strokes has been analysed in detail, comparing the numerical results with the prediction of the theoretical flow rate for non-newtonian fluids in concentric annuli driven by a pressure gradient (Fredickson and Bird [7], Hanks [8]). The different pressure conditions of the pump have been taken into account using different driven velocities for the forward and backward strokes following a sinusoidal law. Hence, the modifications in the aspirating conditions of the pump can be monitored as a function of the cycles per minute imposed. Finally, the unsteady numerical model has allowed the study of the impact of the different piston positions in the internal flow patterns of the grease for every cycle.

\section{GREASE CHARACTERISTICS. LUBRICATING PUMPS AND OPERATING CYCLE}

\section{Greases rheology}

Lubricating greases are, in general, highly-structured colloid dispersions of a thickening component; typically, a metallic soap in a lubricating oil. The most common solution is the use of grease acids of metallic soaps such as lithium, calcium, sodium, aluminium or even barium as thickening elements. The thickening gives consistency to the grease, preventing the fluid leakage or the penetration of contaminant particles, with no penalty on the lubricating properties.

The rheological behaviour of lubricating greases is not a common research area in the literature. This particular application in the lubricating industry covers exclusively a $3 \%$ of all the manufactured lubricating greases worldwide (Delgado et al. [9]). However, a few references can be found in the bibliography concerning the rheological behaviour of greases according to their composition, working temperature or mechanical stress. In particular, it can be cited the work by Delgado et al. [9], where the influence of the thickening soap and the viscosity of the base oil over the grease characteristics is addressed. Other works, like those by Sanchez et al. [10] or Delgado et al. [11-12], deal with the rheological response of lubricating greases with variations in the temperature or in the shear stress.

Nowadays, the greases are standardized using the NLGI classification (National Lubricating Grease Institute, [13]). This method is a well-extended reference in the industry because it simplifies the grease selection only to its overall consistency under normal conditions. The different NLGI grades, from No. 1 to No. 9, determine the consistency of the grease according to a penetration test (ASTM D-217, [14]) where a normalized cone is driven onto a grease test tube to measure its penetration depth after a particular period of time. In the present paper it will be analysed the behaviour of typical NLGI-2 greases in an axial piston pump. This grade presents normalized values of penetration at $25 \circ \mathrm{C}$ between 265 and 295 tenths of millimeter (Rudnick, [15]). 
It is well-known that when a shear force is applied over a portion of grease, a reorientation of the composite fibers is induced and the overall fluidity of the grease improves. To characterize this behaviour, the Ostwald-de Waele model for non-newtonian fluids is taken here (Bird, [16]). This expression implements a non-linear relationship between the shear stress and the velocity gradient, according to a power law as follows:

$$
\mu=k \dot{\gamma}^{m-1} \Rightarrow \tau=\mu\left|\frac{\partial u}{\partial y}\right|=k \dot{\gamma}^{m}
$$

where $k$ and $m$ are both consistency and behaviour indexes respectively, which are determined according to experimental testing.

In this work, it has been considered a NLGI No. 2 grease, with a typical density of $950 \mathrm{~kg} / \mathrm{m}^{3}$ at 20 ㄷ , similar to the commercial grease employed for the characterization of the performance curves of the pump studied here. Both consistency and behaviour indexes have been taken from the values given in Delgado et al. [9], that is, $k=1100$ Pa.s ${ }^{m}$ and $m=0.14$. Figure 1 represents the shear stress and the apparent viscosity as a function of the velocity gradient according to the power law with those coefficients.

\section{Grease pump and operating cycle}

The pump analyzed here is an air-operated, reciprocating piston pump, particularly designed to work with lubricating greases up to grade NLGI-4 by Samoa Industrial S.A., one of the world's leading manufacturers of lubrication equipment. The alternative displacement is driven by a pneumatic motor that inverts the piston direction when it reaches both top and bottom-dead centers respectively (TDC, BDC). This pneumatic cylinder is connected to a set of internal rods that transmit the motion to the hydraulic piston immersed in the tube housing of the pump. The airsupplied pressure exerts a force that is proportional to the area of the pneumatic cylinder, which related to the small area of the hydraulic piston inside the pump leads to a multiplication of the operating pressure in the grease, resulting in an overall ratio of 60:1 (supplied pressure/delivered pressure). This is equivalent to affirm that fixed one particular air-supplied pressure, the number of cycles per minute in the pump will be a function of the pressure drop imposed at the pump discharge, being maximum when the flow is delivered free at the exit (atmospheric outlet pressure) and being zero when the required pressure is higher than the air-supplied pressure times the pressure ratio (typically, 420 bar resulting from 7 bar of the compressed air times the $60: 1$ ratio of the pump). Figure 2 shows a sketch of the pump and how it is usually mounted over the tap of the grease drums.

The grease is pumped in a cycle based on a forward stroke (feeding stage) and a backward stroke (net outflow stage). Figure 3a shows a view of the 3D section of the pump, revealing the aspirating region and the internal labyrinths connecting the working chambers with the outlet ports. The main parts of the pump, numbered in the figure, are the feeder (1), the sleeve bushing acting as a check valve (2), the upper check valve (3), the outlet discharge valve (4) and the pneumatic motor (5).

In a symbolic fashion, figure $3 \mathrm{~b}$ shows a hydraulic sketch of the different pump strokes completed in every cycle, detailing the corresponding valves conditions. During the forward stroke, the feeder injects grease into the pump tube. The pressure is relatively low because there 
is a finite gap within the internal housing that prevents the arising of high pressures in the internal chambers. Hence, the grease is pushed from the primary chamber (1-2), opening the check valve (2) and allowing the grease to be supplied also into the second chamber (2-3). At this stage, the volume of that chamber (light green region) is associated exclusively to the outer diameter of the hydraulic piston. Moreover, this chamber is isolated from the upper passages because of the closure of the check valve (3) which is working due to the pressure inside the upper chamber (3-4). Precisely, that chamber is pressurized according to the closure of the last valve (4), calibrated at the required outlet pressure $\left(p_{c}\right)$. This situation is a consequence of the leakage flow established in the gap between the feeder and the internal diameter of the pump housing, which limits the pressure inside the feeding chamber to just a few bars. Consequently, the advancement of both chambers (1-2) and (2-3) is completed without delivering net mass flow at the pump discharge. Exclusively, when the operating pressure is low enough, there is also an additional supply of lubricating grease in the forward stroke, because the reduced pressure established in the feeding chamber is capable to balance the internal losses and the outlet atmospheric pressure.

Conversely, in the backward stroke, the piston region with a higher diameter $D$ (see the difference between $d$ and $D$ in figure 4) enters progressively into the second chamber (2-3). Because at the end of the preceding stroke, both (2-3) and (3-4) chambers were fully-loaded with lubricating grease, the hydraulic piston pushes the grease volume equivalent to the net difference between both piston sections, thus generating the net volumetric displacement of the pump (barely $5 \mathrm{~cm}^{3}$ for the present pump model). At this moment, the second valve (2) stays closed (just because of the grease pushing the sleeve bushing against the stopper of the check valve), while both valves (3) and (4) are opened (since there is no gap inducing flow leakages, the required pressure is set instantaneously), allowing the lubricating grease to flow outside the pump and establish the net mass flow rate.

Table 1. Geometrical data and operating parameters.

\begin{tabular}{lc}
\hline Pump pressure ratio $[-]$ & $60: 1$ \\
\hline Air motor diameter, $D_{m}[\mathrm{~mm}]$ & 75 \\
\hline Air motor stroke, $L_{m}[\mathrm{~mm}]$ & 75 \\
\hline Outer tube diameter, $T_{d}[\mathrm{~mm}]$ & 30 \\
\hline Inner tube diameter, $t_{d}[\mathrm{~mm}]$ & 23 \\
\hline Hydraulic piston diameter, $D[\mathrm{~mm}]$ & 15 \\
\hline Hydraulic piston stroke, $L[\mathrm{~mm}]$ & 65 \\
\hline Feeder rod diameter, $d[\mathrm{~mm}]$ & 12 \\
\hline Feeder gap, $h[\mathrm{~mm}]$ & 0.2 \\
\hline Pump displacement per cycle $\left[\mathrm{cm}^{3}\right]$ & 4.8 \\
\hline Air-operation pressure, $P_{a}[\mathrm{bar}]$ & $3-7$ \\
\hline Discharge pressure, $\Delta P[\mathrm{bar}]$ & $0-280$ \\
\hline Driven velocity, $n[\mathrm{cycles} / \mathrm{min}]$ & $70-180$ \\
\hline Delivered flow rate,$\dot{m}[\mathrm{gr} / \mathrm{min}]$ & $0-1200$ \\
\hline
\end{tabular}


Figure 4 shows a detail of the pump aspirating region where both operating chambers can be observed in the rendered drawing. The basic geometrical dimensions and all the important mechanical elements have been annotated over a representation of the extreme positions of the piston pump (BDC, TDC). Additionally, all the relevant geometrical data and operating parameters are listed in table 1.

Finally, figure 5 shows the experimental data corresponding to the pump performance, in terms of delivered flow rate as a function of the operating pressure. The figure includes the performance curves for air-supplied pressures of 5, 6 and 7 bar respectively. However, in the present study, only the values corresponding to 7 bar have been used for the validation of the numerical model. Note that, for every required pressure (in ordinates), it corresponds a particular variable driven velocity (in the analyzed range it goes from 70 -maximum pressure- to 180 -minimum pressure- cycles per minute). All the curves present a first region (at high pressure) with a slope that is increased as the supplied pressure rises. Besides, at zero outlet pressure (atmospheric), the delivered flow rate is significantly increased because the feeder of the pump is capable to inject extra lubricating grease also in the forward stroke, as previously discussed.

These experimental curves have been obtained in a normalized test that measured the actual cycles per minute of the pump using a digital tachometer, and the delivered flow rate with a digital flowmeter. The operating pressure was registered with a manometer connected at the pump outlet port, and the required compressed air was also monitored, resulting in typical values in the range of 320 to 650 litres (normal) per minute for all the conditions tested.

\section{NUMERICAL METHODOLOGY}

The commercial package FLUENT v.6.3 [17] has been used to resolve the set of Navier-Stokes equations with a finite volume approach that includes a dynamic mesh technique for the simulation of the alternative displacement of the piston pump. The code, executed unsteadily, predicts the evolution of the flow patterns in the aspirating region as a function of the relative position of the feeder.

\section{Mathematical model}

The finite volume method is employed to resolve the fluid governing equations, transforming a continuum domain into a discretized space of small cells where an algebraic system of equations is resolved after linearization and discretization of the original mathematical equations for the mass and momentum conservation. Considering the present pump geometry, the following basic characteristics have been implemented in the model:

- Full-scale simulation of the pump over a 2D axisymmetric domain where minimum geometrical modifications have been introduced to assure both constructive and operational symmetries of the model.

- Unsteady resolution of the reciprocating piston using the layering method for dynamic meshes.

- Laminar, viscous flow of single-phase, non-newtonian grease, with a pseudoplastic rheological behaviour. 
- Implementation of a parametric UDF to simulate the sinusoidal displacement of the piston pump as a variable function of the stroke and the frequency (in cycles/minute) of the velocity driven by the pneumatic motor.

The temporal discretization employed in this model has been first order, with an upwind spatial scheme for the momentum equation. In spite of being first order, which implies a significant numerical diffusion, the optimal structure used for the mesh (see the discussion about mesh quality afterwards), as well as an accurate selection of reduced time steps, allowed controlling the diffusion for the whole simulation database. The pressure-velocity coupling was resolved using the SIMPLE algorithm and the underrelaxation factors were kept within typical ranges ( 0.3 for the pressure correction equation and 0.7 for the momentum equation), which helped to speed up the convergence process.

\section{Dynamic meshes}

Dynamic and deformable meshes allow the displacement of the domain boundaries in CFD simulations, adjusting the associated mesh to the new locations accordingly. They are used to introduce in the modelling those frontiers that are moving in time (lineally or rotatingly) in the case of positive displacement pumps: alternative engines, gear or vane pumps, axial piston pumps, etc. To introduce this technique, it is necessary to take into account the grid velocity of the boundaries into the general conservation equation (Riemslagh et al., [18]) according to:

$$
\frac{\partial}{\partial t} \int_{V} \rho \phi \mathrm{d} V+\int_{A} \rho \phi\left(\vec{V}-\vec{V}_{g}\right) \cdot \mathrm{d} \vec{A}=\int_{A}(\Gamma \nabla \phi) \cdot \mathrm{d} \vec{A}+\int_{V} S_{\phi} \mathrm{d} V
$$

where $\vec{v}_{g}$ stands for the grid velocity of the dynamic mesh, which is mandatory to evaluate the inlet and outlet flows on the moving boundaries. Moreover, the temporal term in equation (2) must introduce the variation of the cell sizes associated to the moving boundaries overtime. This can be also evaluated from the grid velocity as:

$$
\frac{\partial V}{\partial t}=\int_{A} \vec{V}_{g} \cdot d \vec{A}=\sum_{j=1}^{c . V} \vec{V}_{g, j} \cdot \vec{A}_{j}
$$

Different schemes for the mesh reconstruction can be found in the literature, according to the basic characteristics of the moving mesh. Basically, there are three fundamental methods:

- Smoothing techniques, where the initial connectivity of the mesh nodes is remained unchanged. The nodes are repositioned as if they were connected by small springs. Though there is no restriction about the original mesh type, it can be used only in the case of small deformations.

- Layering techniques, where the creation and/or destruction of cells are completed as the moving boundary advances or returns. It is based on the addition or suppression of cell rows as the moving zone grows or collapses, which implies a change on the topological connectivity of the original mesh. Developed to model the reciprocating displacement of a moving surface (piston), it is restricted for structured meshes because of the requirement of the introduction or elimination of ordered cell rows. 
- Remeshing techniques, which is an alternative to the smoothing algorithm when there are excessively large deformations in the model. The mesh has to be locally rebuilt after the changes in the boundaries are completed.

In the present study, a layering method has been employed for an efficient treatment of the forward and backward strokes of the piston pump. Because in the course of a cycle two consecutive chambers are employed, one depressurized and the other working at high pressure, it was necessary to introduce two different zones of dynamic mesh. The first one, concerning the feeder or primary chamber, defines the movement of the zones adjacent to the feeder (figure 6) which are respectively enlarging or collapsing respect to a couple of interior surfaces defining the piston TDC and BDC. The end zones where the mesh cells are created and destroyed are highlighted in the figure with dashed black lines. In addition, it is necessary to define a sliding interface in the radial gap (dashed yellow line) to allow the transmission of information between the inlet ports of the pump (fixed zone) and the zones adjacent to the feeder, continuously deforming overtime (dynamic zone).

Analogous, in the pressurized chamber, similar zones moving with the feeder accordingly have been defined, responsible to generate the net volume displacement of the pump during the backward stroke (see figure 7).

The velocity of all these zones is imposed with a compiled UDF that associates a sinusoidal law to the displacement of their gravity centers according to $v(t)=A \omega \operatorname{sen}(\omega t)$. The pressurized chamber works against the check valve downstream, so there is no leakage flow during the second stroke. The delivered flow rate is thus only a function of the stroke (two times the amplitude of the sinusoidal law, $A=L / 2$ ) and the frequency (cycles/min of the piston, $\omega=2 \pi f$ ). As a consequence, the instantaneous flow rate can be determined as the flow cross-section $(S)$ times the timeresolved velocity, that is, $Q(t)=S v(t)=S L \omega \operatorname{sen}(\omega t) / 2$, where $L$ represents the piston stroke. The mean flow rate is obtained from the integration of the temporal evolution over one cycle period:

$$
\bar{Q}=\frac{1}{T} \int_{0}^{T / 2} Q(t) d t=L S / T=V_{B} n
$$

\section{Mesh}

A structured mesh with local refinements has been introduced to increase the number of cells in the small gaps within the pump housing, as well as in the aspirating zone, where higher deformation gradients for the grease are expected.

Note that if the mesh is extremely fine, the spatial distributions to be obtained should be of the highest quality, but low time steps and a large number of iterations would be necessary to guarantee convergence. On the contrary, coarse meshes may provide very poor spatial gradients, leading to incorrect predictions and non-realistic solutions.

In the present case, a total number of 25,400 cells has been adopted for the $2 \mathrm{D}$, axisymmetrical domain (see figure 8). Despite of the absence of a systematic analysis regarding grid sensibility, a validation respect to the leakage flow on the feeder gap (where higher gradients are established and local refinement is reinforced) has been considered instead. In particular, with a moderate discretization in the gap (in the order of 8-10 cells to cover the $0.2 \mathrm{~mm}$ height) will be sufficient to 
obtain an accurate prediction of the velocity profile and its associated leakage flow during the forward stage when the pressurized chamber is loaded. This is confirmed in following sections of the paper, where the numerical results for the leakage flow are compared with the theoretical values given for pseudoplastic fluids in concentric annuli.

Nevertheless, it is more important to assure that the delivered flow rate at the discharge exit as well as the operating pressure inside the pump are correct, rather than a very precise capture of the leakage flow phenomena. Due to the constructive characteristics of the pump, as well as the driven mechanism, it can be easily checked that the prediction of these variables is independent of the mesh density employed. This assertation is based on the following particularities:

- The delivered flow rate is guaranteed because of the piston effect (volumetric), without any leakage, in the second chamber. Thus, it is completely independent of the spatial discretization (zero order) used in the internal gaps of the machine, in both the first chamber and the adjacent labyrinths.

- The operating pressure fixes the actual driven velocity of the pneumatic motor. Because in the simulation both velocity and outlet pressure are imposed as an operational parameter and an outlet boundary condition respectively, the working point is assured.

In fact, the simulation allows the estimation of the internal pressure drop in the pump, and predicts the mechanical torque required for the air-operated motor to work at the discharge pressure at a particular alternative velocity. The discretization adopted close to the solid walls is essential for an accurate estimation of the friction on the walls (function of the grease shear stress and the flow velocity gradients) and, therefore, the mechanical power required. However, this parameter is less critical in the present case, because the real interest of the simulation is the analysis of the aspirating zone, rather than the study of shear flows in the internal passages.

Furthermore, the extension in the range of variation of the grease viscosity with the velocity gradient (see figure 1), implies that real changes in the flow patterns are relevant only when the order of magnitude in the gradients is modified. This means that a good estimation of the shear stress (imposed by the rheological behaviour of the fluid) can be obtained with a moderate number of cells in the internal gaps, assuring an accurate numerical simulation even with a relatively coarse mesh.

\section{Boundary conditions}

The different boundary conditions introduced in the model are also represented in figure 8. A horizontal inlet pressure condition (atmospheric) has been defined for the grease free surface in the drum. This kind of condition is better posed when it is assigned over a horizontal plane to fit a constant pressure value. Regarding the solid boundaries of the pump housing, all of them have been defined as no-slip endwalls. Respect to the moving zones, the walls are also defined stationary but in the relative reference frame.

To complete a correct simulation of both forward and backward strokes, a set of different boundary conditions for the outlet port and the internal check valve (2) are applied according to each stage of the cycle: 
- During the piston rising, the outlet is sealed fixing a wall boundary condition which simulates the closure of the check valve. However, in the returning, because the sleeve bushing is blocked, there is no leakage flow and the outlet pressure equalizes the required pressure of the system, so an outlet static pressure is imposed as the boundary condition in the backward stroke.

- Analogous, in the sleeve bushing zone, it is necessary to switch the condition: either flow rate must be allowed to pass, or must be blocked. In the real pump, the small piece (see detail in figure $8 \mathrm{~b}$ ) is displaced by the fluid, connecting both chambers or isolating the passage. In order to avoid an extra complexity for this modelling (the small piece should be defined with a 6-DOF algorithm, to be moved by the balance of inertial and viscous forces exerted by the circulating fluid), the sleeve bushing was fixed to its central position (the real limitation, represented by the small gap of $1 \mathrm{~mm}$ between the piece and the outer casing is maintained) and the passage is opened or closed pending on the cycle stage. Hence, in the rising, the passage is opened imposing an interior boundary condition, while in the returning, it is switched to an internal wall, blocking the flow pass.

\section{Numerical procedure and stability}

The resolution of the numerical model is initialized resolving the flow through the pump when the piston is located at BDC (see figure 8). Afterwards, a complete cycle is executed unsteadily, calculating both forward and backward strokes, in order to stabilize the starting-up transient. Because the model is single-phase, it is assumed that the pump is fully-loaded with grease from the beginning of the simulation, so the duration of this initial transition is minimized. Following, a new second cycle is executed and all the variables of interest (pressure, velocity and viscosity maps) are stored in the course of that revolution. Besides, the pressure and corresponding flow rates in the different pump chambers are monitored in order to perform a complete unsteady post-processing for the later results section. When reaching both TDC and BDC, it is mandatory to change the outlet condition for the pressure and the inner restriction for the flow pass as explained above.

Four different operating conditions of the pump have been executed for the numerical database, corresponding to the following driven velocities: 70, 100, 160 and 180 cycles/min. See table 2 below with a summary of the more important operational parameters of this simulation set. The operating pressure for the backward stroke has been taken according to the experimental data given by the manufacturer (see figure 5). As it can be seen in the figure, the different cases correspond to high (280 bar), normal (232 bar), low (112 bar) pressures, as well as the free delivery condition ( 0 bar).

To enhance the comparison of temporal results, a variable time step was fixed for the different simulations, so the total number of time steps during the course of a cycle would be the same for all the cases. In other words, each particular intermediate position for the piston displacement would be reach after the same number of time steps in every simulation. In particular, 1000 time steps were fixed to cover every cycle, resulting obviously in 500 time steps for the forward and another 500 for the backward stroke. Note that with the driven velocities of this application (table 2 ), it is necessary to fix typical time steps in the order of $10^{-4}$ seconds (from 3.33 to $8.57 \times 10^{-4} \mathrm{~s}$ ). 
To preserve the stability and the convergence of the dynamic algorithm is necessary to fulfil that the displacement of every moving cell per time step must be lower than its characteristic cell size. The objective is avoiding that the simulation could advance per time step a larger distance than that defined by the longitudinal cell size. This restriction is equivalent to the Courant number employed for classical unsteady simulations with explicit schemes (also known as CourantFriedrichs-Levy condition, or CFL - Hirsch [19]), that can be expressed as $C F L=v \Delta t / \Delta x$, which guarantees numerical stability if the parameter is lower than unity $(C F L<1)$. Considering the characteristic velocity for the CFL number as the maximum velocity of the axial piston (see table 2) at midstage of every stroke, and a typical cell size of $0.25 \mathrm{~mm}$ (longitudinal size within the feeder zone), a CFL value of $0.816(<1)$ is obtained for all the cases, which meets with the specified criterion.

Every unsteady simulation required approximately $4 \mathrm{~h}$ of CPU time, all of them executed on a single PC IntelCore(Duo), double-core processer, $3 \mathrm{GHz}$, and $4 \mathrm{~Gb}$ RAM. Also, 50 iterations were fixed per time step, so minimum residuals were maintained for both continuity (in the range of $\left.10^{-4}\right)$ and momentum $\left(10^{-5}\right)$ equations, matching by far the usual convergence criteria.

Table 2. Numerical database and basic operating parameters.

\begin{tabular}{|c|c|c|c|c|c|c|c|}
\hline Operating point & $\begin{array}{c}n \\
(c y c / m i n)\end{array}$ & $\begin{array}{c}\Delta P \\
\text { (bar) }\end{array}$ & $f(H z)$ & $T(s)$ & $\begin{array}{l}\Delta t(s) \\
\times 10^{-4} \\
\end{array}$ & $\begin{array}{l}V_{\max } \\
(\mathrm{m} / \mathrm{s})\end{array}$ & $\begin{array}{c}\text { CFL } \\
\text { number }\end{array}$ \\
\hline High pressure & 70 & 280 & 1.166 & 0.857 & 8.57 & 0.238 & 0.816 \\
\hline Normal pressure & 100 & 232 & 1.666 & 0.600 & 6.00 & 0.340 & 0.816 \\
\hline Low presssure & 160 & 112 & 2.666 & 0.375 & 3.75 & 0.544 & 0.816 \\
\hline Free delivery & 180 & 0 & 3.000 & 0.333 & 3.33 & 0.612 & 0.816 \\
\hline
\end{tabular}

\section{RESULTS ANS DISCUSSION}

Following, the results obtained from the post-processing of the FLUENT simulation are shown. All the cases considered, summarized in table 2, have allowed the analysis of the whole operating range of the pump when supplied with compressed air at 7 bar. Firstly, the variations of pressure and velocity fields with the piston stroke are observed for the reference simulation (normal pressure, 232 bar, at $n=100$ cycles/min), in order to check that the numerical description of the operating cycle is optimal. Besides, global variables, such as the delivered flow rate or the pressure in both chambers, are analyzed to illustrate the flow patterns within the pump as a function of the operating pressure. Locally, the leakage flow in the feeder has been plotted to validate the spatial discretization of the gaps. Finally, a detailed analysis of the grease viscosity maps, both inside and outside the pump, has been carried out to predict the fluidity conditions of the grease for the aspirating ports of the machine.

\section{Simulation of the operating cycle}

Figure 9a shows the evolution of the pressure and flow delivered with the piston displacement for the reference situation (100 cycles/min). In the abscissa, the longitudinal position has been adimensionalized with the hydraulic piston stroke, presenting a range that goes from 0 to 2 . 
During the forward stroke $(0<x / L<1)$, there is no flow delivered, and the second chamber remains depressurized. Meanwhile, in the first chamber, the pressure evolves from a slight depression to a moderate 2 bar overpressure when the feeder passes the inlet ports and gets into the housing of the pump (aprox. at $x / L=0.25)$. During the backward stroke $(1<x / L<2)$, the returning of the piston provokes a depression in the feeding chamber (until $x / L<1.75$ ) that disappears when the feeder gets again into the inlet ports. Obviously, the absolute value around three negative bar is physically unfeasible because highest depression cannot be over 1 atmosphere when vacuum is generated. In the real process, partial cavitation and break-down of the grease continuity must overcome earlier, compromising seriously the mechanical stability and operation of the feeder.

On the other hand, the second chamber is set to the outlet pressure (in this case, 232 bar) and the delivered flow rate presents a perfect sinusoidal evolution due to the alternative displacement of the driven motor and the lack of any leakage flow. The area under the curve provides the mean flow rate per cycle, $393 \mathrm{gr} / \mathrm{min}$ for the reference case. In addition, figures $9 \mathrm{~b}$ and $9 \mathrm{c}$ reveal the pressure field (A) and the velocity vectors (B) for the backward stroke $(x / L=1.5)$ when the instantaneous flow rate is maximum. The pressurized chamber is all set to 232 bar, according to the Pascal principle for an incompressible flow. Analogous, the representation of the velocity vectors shows how the piston (inducing the net displacement due to the difference between the inner and outer diameters $d$ and $D$ ) establishes a relative net flow within the stem of the piston, going upwards to the pump outlet. Maximum velocities, in the range within $1 \mathrm{~m} / \mathrm{s}$, are similar to those followed by the piston during the strokes.

\section{Comparison between operating points}

The evolutions of the pressure and flow rate inside the pump overtime are compared now for all the cases tested. Figure 10 illustrates the pressure in the first chamber. When the driven velocity is increased, both depressions and overpressures in backward and forward strokes are respectively enlarged. The unphysical negative values of depression provided by the numerical approach are a consequence of the single-phase simulation adopted here. In order to reproduce correctly the inception of cavitation and the break-down of the grease continuity during the returning, it is necessary to introduce a two-phase simulation (air-grease) with an algorithm to take into account cavitation phenomena (volume fraction of trapped air in the grease). The evolution of the pressure in the second chamber has not been shown here, because it gives only a uniform value of the pressure attained at the outlet port for every case (as already shown in the midplot of figure 9a).

Finally, figure 11 shows the envelope of the sinusoidal laws as the frequency of the stroke increases. According to equation (3), a higher number of cycles per minute imply higher amplitude of the sine wave and thus a larger delivered flow rate. Note that at free delivery ( $n=180$ cycles/min) there is also some amount of extra flow rate during the forward stroke, just after the feeder passes by the inlet ports and enters into the tube housing. In this representation, it has been plotted as negative (bottom quadrant) because of the opposite direction of the piston displacement during the forward stroke respect to the returning stage, but it must be accounted also as delivered flow rate for the operating cycle.

The integration of all the curves represented in the previous figure provides the mean flow rate to be compared with the experimental curves (see figure 12). Quite remarkable is the difference for the free delivery conditions, where the experimental results provide a reference value of 1130 
$\mathrm{gr} / \mathrm{min}$, while the simulation estimates only $937 \mathrm{gr} / \mathrm{min}$, that is, a $16 \%$ discrepancy. A possible explanation to this difference could lay on the mismatch between the real stroke of the pump (close to the $75 \mathrm{~mm}$ of the air-operated cylinder) and the stroke considered in the simulation (just $65 \mathrm{~mm}$ ). When the required pressure is very low, the mechanical stoppers of the pump are relaxed, allowing the effective stroke of the axial piston to be close to the stroke of the air motor.

\section{Leakage flow in the feeder}

To validate the discretization of the model, as well as to evaluate the recirculation in the internal gaps, the velocity distribution in the feeder gap has been monitored as a function of the pressure drop overtime. In order to illustrate how the leakage flow is established in both forward and backward strokes, velocity vectors in the gap have been superimposed to the pressure field at both sides of the feeder in figures 13 and 14. The tangential shear induced by the feeder plate generates an additional velocity profile, opposite to the pressure gradient, but it results one order of magnitude lower than the one derived from the driven pressure. Hence, the effect of the moving surface (Stokes flow) can be neglected respect to the pressure effect (Couette flow) for this situation (see the figures).

The values of pressure, $\Delta p 1$, monitored in the right-hand side of the feeder overtime for figure 10 , have been reutilized to compute the theoretical expression of the flow rate for a nonnewtonian fluid in concentric annuli driven by a pressure gradient (Hanks and Larsen, [20]). Because of the complexity of the rheological power law, some authors have developed explicit approximations to evaluate the flow rate as a direct function of the pressure drop without calculating the intermediate location of maximum stress (Bird, [16]). In particular, we have employed the formulation given by David and Filip [21] to estimate the leakage flow as:

$$
Q_{t h}=\frac{\pi R^{3} m}{2(1+2 m)}(1-\kappa)^{2+1 / m}(1+\kappa)\left(\frac{\Delta p^{*} R}{2 k}\right)^{1 / m}
$$

where $k$ and $m$ are the rheological parameters, $\Delta p^{*}=\Delta p / /$ is the reduced pressure gradient, $R$ is the mean radius of the passage and $\kappa=1-h / R$, being $h$ the radial gap. Figure 15 shows the comparison between the flow rates obtained numerically (dots) and the theoretical evolution according to (5) as a function of the pressure $\Delta p 1$ monitored in the primary chamber (dashed lines). Note the overall agreement between the predictions and the coherency of the results that confirm higher leakage flow during the returning (higher depression in that stage) and how it goes to zero at BDC.

\section{Viscosity maps and fluidity zones}

To conclude the paper, viscosity maps in both interior regions of the pump and close to the aspirating ports within the grease drum are shown below. Unsteady results for the reference operating conditions as well as a comparison between all the simulated cases for the more relevant intermediate positions are presented to analyze the impact of the different operational parameters.

Due to the higher gradients observed in the interior gaps, the maps representing viscosity distributions inside the pump are drawn using a colour scale ranging from 0.01 to 200 Pa.s; while in the outer locations within the drum, the scale is extended to $10,000 \mathrm{~Pa}$.s to show regions with 
zero fluidity. Note that these scales have been saturated, so all the red zones indicate regions where the dynamic viscosity is, at least, equal to the maximum of that scales.

Figure 16 represents viscosity maps overtime inside the pump, for both forward and backward strokes of the normal pressure operation. At both BDC and TDC, the piston zero velocity makes disappear the grease velocity gradients, increasing the dynamic viscosity drastically (the grease cannot flow). As expected, maximum velocities are increasing the grease fluidity at mid-strokes, which enhances the loading of the primary chamber of the pump. It can be observed that the fluid is moving in a sort of slug flow where "solid packages" of grease are transported between the moving boundaries and internal gaps that present higher gradients and thus higher fluidity (see the internal labyrinths between adjacent chambers at $0.125<t / T<0.375$ ). In essence, the grease slides over the moving boundaries of the small gaps, inducing the injection of the grease solid portions trapped inside. During the forward stroke, the depression in the first chamber generates a significant influence zone at the inlet, improving the aspirating conditions. This effect is also observed in the second chamber when the net displacement volume is created (see the final endright zone at $t / T=0.125$ and $t / T=0.250)$. In the backward stroke, the first chamber is not experiencing so evident fluidity conditions, which indicates the absence of a significant grease backflow into the drum. On the contrary, it is more remarkable the grease injection due to the piston effect and the major fluidity close to the wall shear layers where the grease is sliding. In the central regions, solid packages of grease are created and injected progressively towards the pump discharge.

Complementarily, figure 17 shows similar results, but now focused on the aspirating region. Once again, only those zones coloured in dark blue (around 100-200 Pa.s) present some fluidity for the grease. In the midstroke position, relative low viscosity regions are developed within the drum. These zones are extended towards the lateral endwalls of the barrel, repeating the wellknown pattern of low viscosity associated to higher velocity gradients. The grease slides over the lateral drum walls, so the central bulk region of grease slips towards the aspirating region to cover the volume left by the aspirated packages close to the inlet ports. During the returning, this flow pattern is repeated, but with a reduced affection zone (compare the maps for $t / T=0.250$ and $t / T=0.750$ to observe that the backflow is quite weak).

These results reveal that the high apparent viscosity for this type of greases (in the range of $10^{3}$ Pa.s) compromises very seriously the aspirating conditions of these pumps. Only in the vicinity of solid walls or in the small inner gaps where notable velocity gradients can be established it is possible for the grease to present certain fluidity (dark blue regions in figures 16 and 17). However, in the inlet ports, quite far from the feeder gap, the negative 1 bar depression generated by the piston effect is the only favourable factor inducing the pump loading. Therefore, with such data for those zones affected by an adverse pressure gradient, it is not evident to define neither the minimum fluidity conditions for the grease, in terms of dynamic viscosity, nor the drum zones really affected by the pump suction.

To prevent an order of magnitude relating all these variables, which also could provide a better interpretation of the numerical results obtained, an inspectional analysis over the governing equations in the aspirating region has been attempted. Considering the symmetry of the problem and simplifying for steady flow, obviating the gravitational forces and imposing the limit fluidity conditions (equalizing the pressure gradient, as the unique favourable force, with the viscous shear stress, as the motion resistance), the following relationship is attained: 


$$
\frac{\partial p}{\partial x} \sim \frac{\partial \tau}{\partial y} \Rightarrow \frac{\Delta p}{L_{x}} \sim \frac{\tau}{L_{y}}
$$

And introducing the rheological definition of the grease, taking into account that it can be approximated (at least in order of magnitude) to a Bingham plastic with an extremely high apparent viscosity (a linear evolution for the region of fluidity), it yields:

$$
\tau=\mu \dot{\gamma}=\mu \frac{\partial u}{\partial y} \sim \mu \frac{v}{h} \quad \text { where } \quad v \sim \frac{Q}{S}=\frac{Q}{L_{y} h}
$$

Note that in the definition of the velocity as a function of the flow rate, it has been considered that the crossed-area for this kind of volumetric pumps is extremely thin (gap with a characteristic height $h$ ), so the section can be approximated to the product of the radial characteristic length (in this case, the pump diameter) times the small gap. Finally, combining (6) and (7), and reordering to obtain an expression of the characteristic lengths as a function of the other parameters, it gives:

$$
\frac{L_{x}}{L_{y}^{2}} \sim \frac{\Delta p}{\mu Q} h^{2}
$$

where $L_{x}$ represents the longitudinal distance where the suction $\Delta p$ induced at the inlet of a pump with a characteristic size, $L_{y}$ (for instance, the diameter $D$ ), and a small gap $h$, is capable to generate a flow rate $Q$ of grease with an apparent viscosity $\mu$.

Substituting with the typical values in S.I. units, $h \sim 10^{-3}(1 \mathrm{~mm}), L_{y}=D \sim 10^{-2}(10 \mathrm{~mm}), \Delta p \sim 10^{5}$ (1 bar), $Q \sim 10^{-5}$ (600 gr/min) and $\mu \sim 10^{2}$ (100 Pa.s, similar to a ketchup's viscosity), it is obtained $L_{x} \sim 10^{-2}(1 \mathrm{~cm})$, that is, an aspirating region where the grease is flowing in the order of the centimeter. Note that if the viscosity is increased one order of magnitude (reaching up to $10^{3}$ Pa.s), the distance $L_{x}$ is dropped also one order of magnitude, reducing drastically the characteristic length to just $1 \mathrm{~mm}$.

The numerical results shown above confirm these rough estimations from the dimensional analysis. The viscosity is lower than 500 Pa.s only in the vicinity of the aspirating ports of the pump, increasing rapidly as we move towards the external zones of the drum (with a characteristic diameter of $50 \mathrm{~mm}$ in this case). There, the grease viscosity is two orders of magnitude higher, practically solidified, and sliding over the endwalls in a sort of highly-viscous packages.

To conclude this section, the impact of the pump cycles/minute on the viscosity maps is also analyzed. At first sight, it is presumable that a higher piston velocity could involve higher gradients enhancing the grease fluidity, at least in the internal passages. Figure 18 compares, for the maximum piston velocity, the viscosity conditions within the pump as a function of the cycles/min employed. It can be clearly observed a higher fluidity where high velocities are developed, especially in the inlet suction and ahead of the axial piston.

Finally, the results inside the drum are also shown in figure 19, comparing all the cases for intermediate positions close to both TDC and $\operatorname{BDC}(x / L=0.75$ and $x / L=1.75$, respectively). In this representation, with a higher scale range, it is revealed the major influence of higher driven 
velocities in the aspirating zone. In particular, the direct comparison of the highest ( $180 \mathrm{cycle} / \mathrm{min}$ ) with the lowest $(70 \mathrm{cycle} / \mathrm{min}$ ) velocities shows the differences respect to how the grease slides over the drum external wall. In this figure, it can be also observed how the central region of packaged grease (on top of the drum) is significantly larger during the returning than in the advancement stroke. On the contrary, the backflow towards the grease drum during the backward stroke provokes that bottom regions in the drum are more fluid that during the forward stage.

\section{CONCLUSIONS}

An unsteady numerical simulation of the non-newtonian flow for a pseudoplatic grease NLGI-2 inside an axial piston pump has been developed using dynamic meshes with a layering algorithm. In particular, the aspirating conditions of the pump have been analyzed in detail for both forward and backward strokes, as a function of the driven velocity of the axial piston, imposed by the operating pressure of the lubricating system.

The validation of the numerical model has been carried out through the comparison of the performance curves, provided by the manufacturer, with the numerical results concerning pressure and flow rate delivered. In addition, the leakage flow in the primary chamber has been contrasted with the theoretical estimations for pseudoplastic, power-law fluids, driven by pressure gradients in concentric annuli.

The numerical model confirms the operational evidences respect to the extremely low fluidity of the grease and the significant limitation that this effect provokes in the aspirating zone. The represented viscosity maps, either as a function of the piston displacement or the driven velocity, reveal highly-pulsating flow patterns of grease, where a sort of slug flow is established with solid packages of grease sliding between the moving walls and the internal gaps. This is due to the rheological behaviour of the grease, which fluidity is enhanced close to the solid walls where velocity gradients arise. Therefore, the grease flows towards the aspiration sliding over the lateral drum walls and creating cavities that the bulk solid packages must occupy during the slow descend of the lubricating. Therefore, a major presence of solid walls, for instance introducing transversal baffles inside the drum could improve the grease injection towards the inlet ports of the pump.

The inspectional analysis of the grease governing equations in the aspirating region, derived from the definition of relevant parameters and characteristic lengths of the flow (in terms of order or magnitude for gaps, diameters and influence zones of fluidity) has provided a better interpretation of the numerical results and a complementary view of the grease condition. Hence, the values obtained via simulation have been confirmed, using an alternative tool to determine the real fluidity of the aspirating grease.

The collection of results presented here is a valuable first step for the numerical modelling of this type of volumetric pumps for lubricating greases, up to now unexplored to the authors' knowledge. It has been evidenced the geometric complexity of the designs and the difficulty associated to the definition of dynamic meshes for an accurate unsteady simulation of this type of pumps. However, it has been possible to develop a robust and operative model, capable to provide overall good results in terms of global performance for the pump. In addition, the limitations of a single-phase model have been also advised, unable to model correctly the inception of cavitation or the continuum break-down due to the pump suction in the backward 
stroke. In the near future, it will be necessary to introduce a two-phase algorithm in the model for a correct treatment of cavitation phenomena that could reproduce all the complexity of the internal flow in the reciprocating axial pump.

\section{ACKNOWLEDGEMENTS}

The authors acknowledge the technical advice given by the Research and Investigation Staff of Samoa Industrial S.A. during the analysis and modeling stages of the axial piston pump, and the technical data provided regarding pump dimensions and performance curves.

\section{NOMENCLATURE}

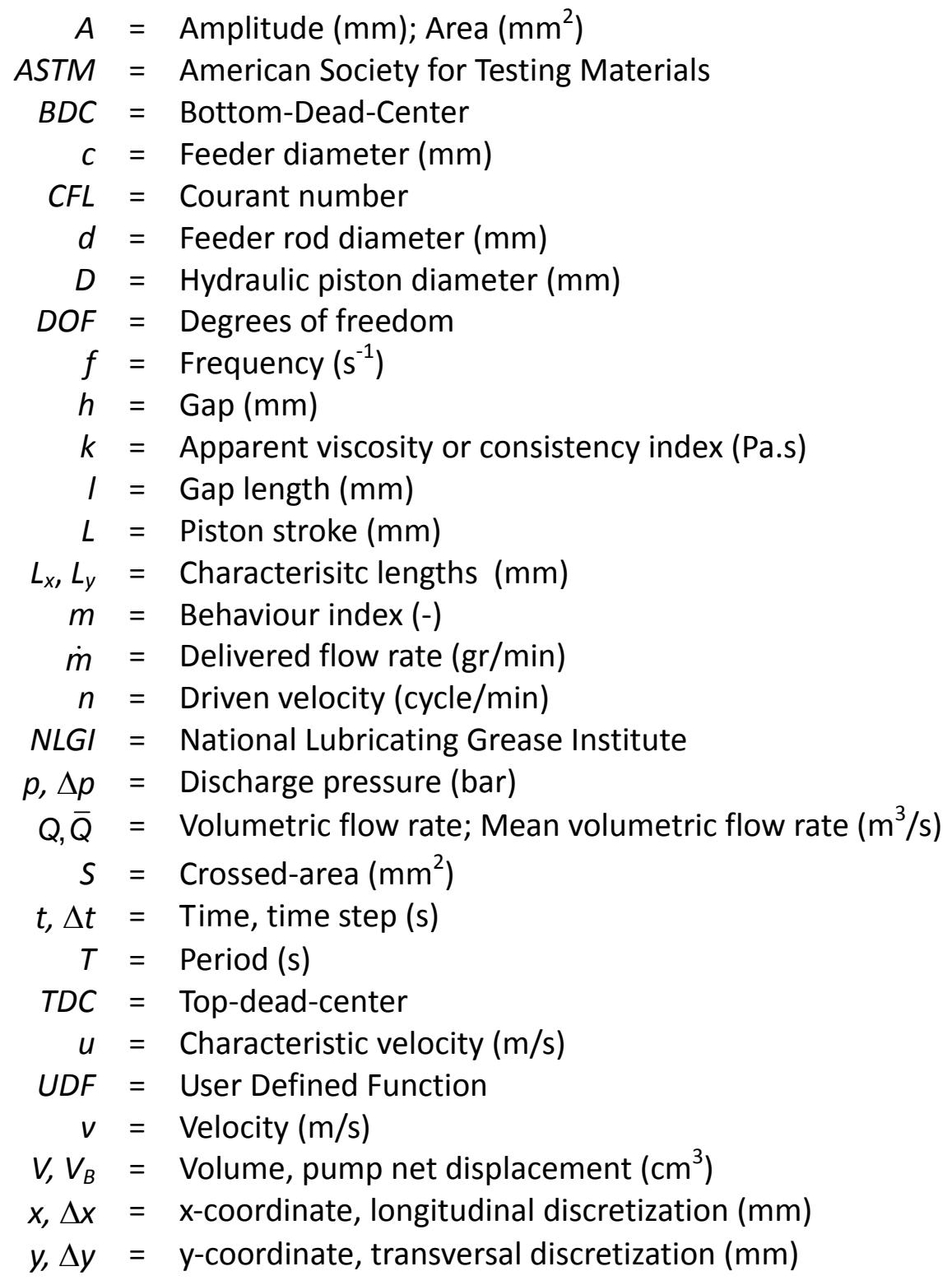

\section{Greek symbols}

$\phi=$ Generic variable 


$$
\begin{aligned}
\mu & =\text { Dynamic viscosity }(\text { Pa.s) } \\
\gamma & =\text { Velocity gradient }\left(\mathrm{s}^{-1}\right) \\
\rho & =\text { Density }\left(\mathrm{kg} / \mathrm{m}^{3}\right) \\
\omega & =\text { Rotational speed }(\mathrm{rpm}) \\
\kappa & =\text { Internal gap distance }(\%) \\
\tau & =\text { Shear stress }(\mathrm{Pa}) \\
\Gamma & =\text { Diffusion coefficient }\left(\mathrm{m}^{2} / \mathrm{s}\right)
\end{aligned}
$$

\section{REFERENCES}

[1] Houzeaux, G; Codina, R. "A Finite Element Method for the Solution of Rotary Pumps". Computers \& Fluids (2007), vol. 36, pp. 667-679.

[2] Vande Voorde, J.; Vierendeels, J.; Dick, E. "Flow simulations in rotary volumetric pumps and compressors with the fictitious domain method". Journal of Computational and Applied Mathematics (2004), vol. 168, pp. 491-499.

[3] Riemslagh, K.; Vierendeels, J.; Dick, E. “An arbitrary Lagragian-Eulerian finite-volume method for the solution of rotary displacement pump flow". Applied Numerical Mathematics (2000), vol. 32, pp. 419-432.

[4] Strasser, W. "CFD Investigation of Gear Pump Mixing Using Deforming/Agglomerating Mesh". ASME Journal of Fluids Engineering (2007), vol. 129, pp. 476-484.

[5] Kumar, S.; Bergada, J.M.; Watton, J. "Axial piston pump grooved slipper analysis by CFD simulation of three-dimensional NVS equation in cylindrical coordinates". Computers \& Fluids (2009), vol. 38, pp. 648-663.

[6] Hyun, K.; Hazel, M.; Suresh, P. "Two-dimensional CFD Analysis of a Hydraulic Gear Pump". Proceedings of the 2007 American Society of Engineering Education Conference (Louisville, Kentucky), 1-3 abril (2007). Paper No. AC2007-821.

[7] Fredickson, A.G.; Bird, R.B. "Non-Newtonian Flow in Annuli". Ind Eng. Chem. (1958), vol. 50, pp. 347-352.

[8] Hanks, R.W. "The Axial Laminar Flow of Yield-Pseudoplastic Fluids in a Concentric Annulus". Ind. Eng. Chem. Process Des. Dev. (1979), vol. 18(3), pp. 488-493.

[9] Delgado, M.A.; Valencia, C.; Sánchez, M.C.; Franco, J.M.; Gallegos, C. "Influence of Soap Concentration and Oil Viscosity on the Rheology and Microstructure of Lubricating Greases". Ind. Eng. Chem. Res. (2006), vol. 45, pp. 1902-1910.

[10] Sánchez, M.C.; Franco, J.M.; Valencia, C.; Gallegos, C.; Urquiola, F.; Urchegui, R. "Atomic Force Microscopy and Thermo-Rheological Characterization of Lubricating Greases". Tribology Letters (2010), vol. 41, pp. 463-470.

[11] Delgado, M.A.; Valencia, C.; Sánchez, M.C.; Franco, J.M.; Gallegos, C. "Thermorheological behaviour of a lithium lubricating grease". Tribology Letters (2006), vol.23(1), pp. 47-54.

[12] Delgado, M.A.; Franco, J.M.; Valencia, C.; Kuhn, E.; Gallegos, C. "Transient shear flow of model lithium lubricating greases". Mech. Time-Depend. Mater. (2009), vol. 13, pp. 63-80.

[13] “NLGI, Lubricating Greases Guide”, National Lubricating Grease Institute, Kansas, USA (1994).

[14] "Standard Test Methods for Cone Penetration of Lubricating Grease" - ASTM D217-10 (2010). 
[15] Rudnick, L.R. "Synthetics, Mineral Oils, and Bio-Based Lubricants: Chemistry and Technology (Chemical Industries)". Ed. CRC (2005), pp. 468.

[16] Bird, R.B.; Stewart, W.E.; Lightfoot, E.N. "Transport Phenomena", Chapter 2. Ed. Wiley (1965).

[17] "Fluent 6.3 User's guide" - Fluent Inc. (2006).

[18] Riemslagh, K.; Vierendeels, J.; Dick, E. "Simulation of Incompressible Flow in Moving Geometries" - VKI Lecture Series (1998).

[19] Hirsch, C. "Numerical Computation of Internal and External Flows: The Fundamentals of Computational Fluid Dynamics". Ed. Elsevier-Butterworth-Heinemann (2007).

[20] Hanks, R.W.; Larsen, K.M., "The Flow of Power-Law Non-Newtonian Fluids in Concentric Annuli". Ind. Eng. Chem. Fundam. (1979), vol. 18(1), pp. 33-35.

[21] David, J.; Filip, P., "Explicit pressure drop-flow rate relation for laminar axial flow of power-law fluids in concentric annuli". Journal of Petroleum Science and Engineering (1996), vol. 16, pp. 203-208.

\section{LIST OF FIGURES}

Fig. 1. Viscosity and shear stress as a function of the velocity gradient for a non-newtonian NLGI-2 grease.

Fig. 2. Sketch of the grease pump. Arrangement over the drum tap.

Fig. 3. a) Rendered 3D view of the axial piston pump. b) Hydraulic sketch of the pump operation.

Fig. 4. Geometry of the pump aspirating region.

Fig. 5. Experimental performance curves provided by Samoa Industrial S.A.

Fig. 6. Definition of dynamic meshes for the primary chamber (feeder).

Fig. 7. Definition of dynamic meshes for the secondary chamber (high-pressure zone).

Fig. 8. Pump mesh. a) Extreme locations of the pump stroke (BDC, TDC). b) Mesh details for the sleeve bushing and the feeder gap.

Fig. 9. Operating variables for the reference case $(n=100 \mathrm{cyc} / \mathrm{min})$. a) Pressure evolution on primary, $\Delta p 1$, and secondary, $\Delta p 2$, chambers, as well as delivered flow rate as a function of the piston stroke. b) Pressure distribution in the pressurized chamber, $\Delta p 2$ [bar], at TDC, $x / L=1.5$. c) Velocity vectors in the pressurized chamber $[\mathrm{m} / \mathrm{s}]$, at TDC, $x / L=1.5$.

Fig. 10. Evolution of the pressure in the primary chamber overtime for all the tested cases.

Fig. 11. Evolution of the delivered flow rate overtime for all the tested cases.

Fig. 12. Numerical-experimental comparison of the pump performance curves.

Fig. 13. Pressure distribution and velocity vectors in the feeder gap during the forward stroke $(x / L=0.5)$ for the reference case $(n=100 \mathrm{cyc} / \mathrm{min})$.

Fig. 14. Pressure distribution and velocity vectors in the feeder gap during the backward stroke $(x / L=1.5)$ for the reference case $(n=100 \mathrm{cyc} / \mathrm{min})$.

Fig. 15. Numerical-theoretical comparison of the leakage flow through the feeder gap as a function of the piston displacement and the operating pressure. 
Fig. 16. Evolution of the dynamic viscosity inside the pump in the course of an operating cycle (results from the reference case, $n=100 \mathrm{cyc} / \mathrm{min}$ ).

Fig. 17. Evolution of the dynamic viscosity in the aspirating region in the course of an operating cycle (results from the reference case, $n=100 \mathrm{cyc} / \mathrm{min}$ ).

Fig. 18. Contours of dynamic viscosity inside the pump for the different operating conditions (results at the maximum fluidity instant).

Fig. 19. Contours of dynamic viscosity in the aspirating region for the different operating conditions (results at the maximum fluidity instant). 


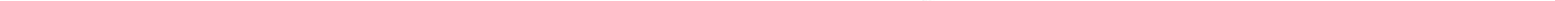



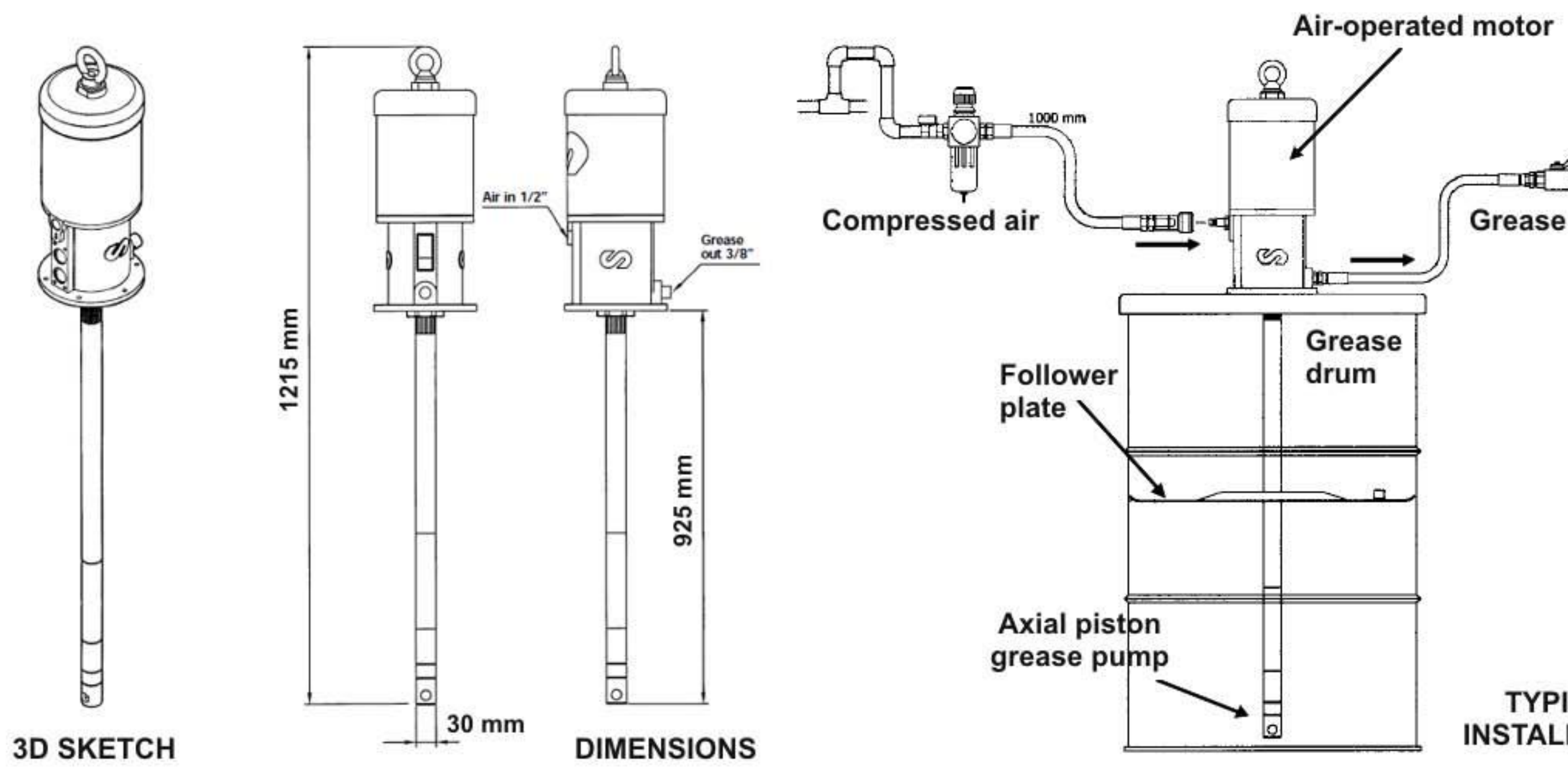

TYPICAL INSTALLATION 


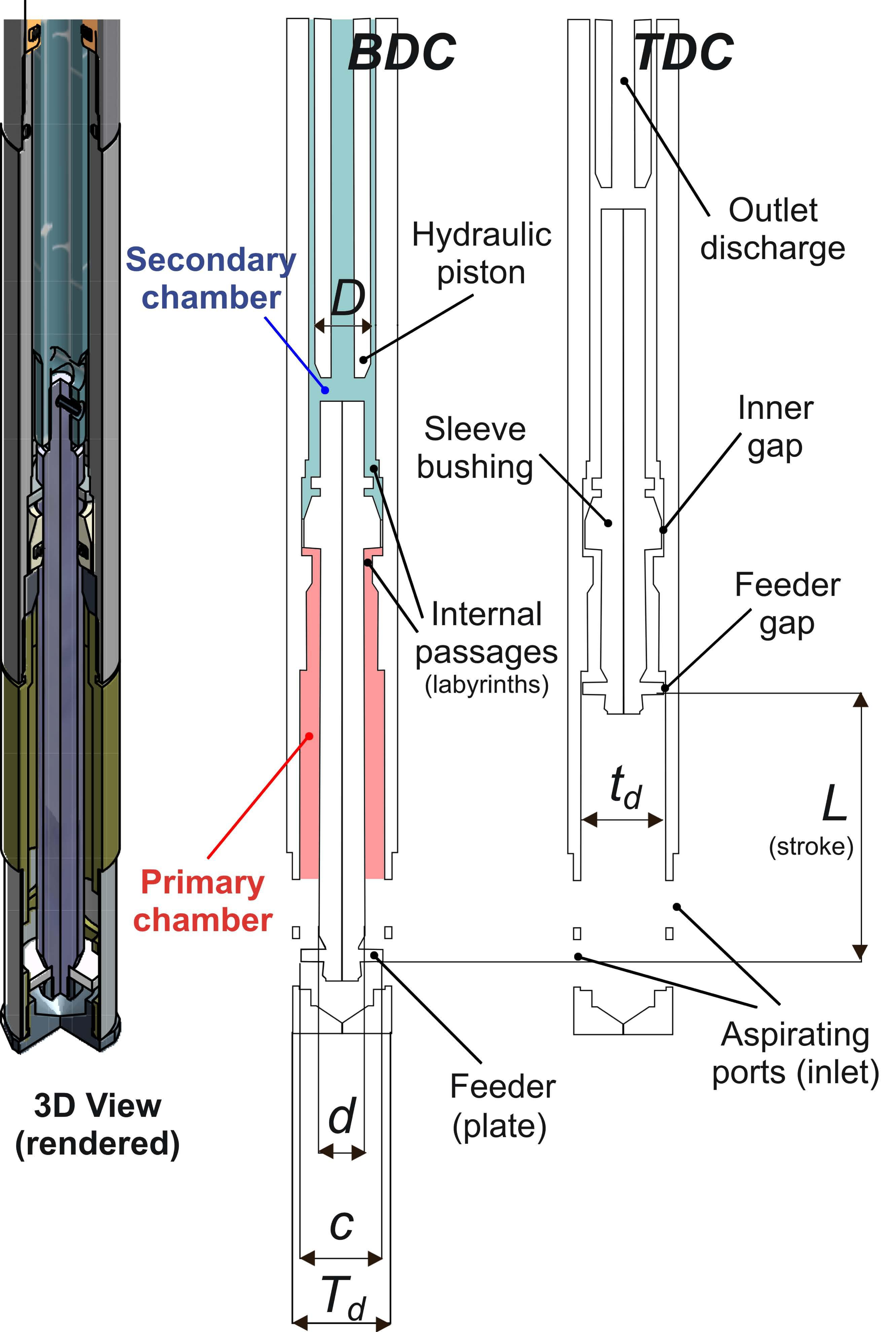




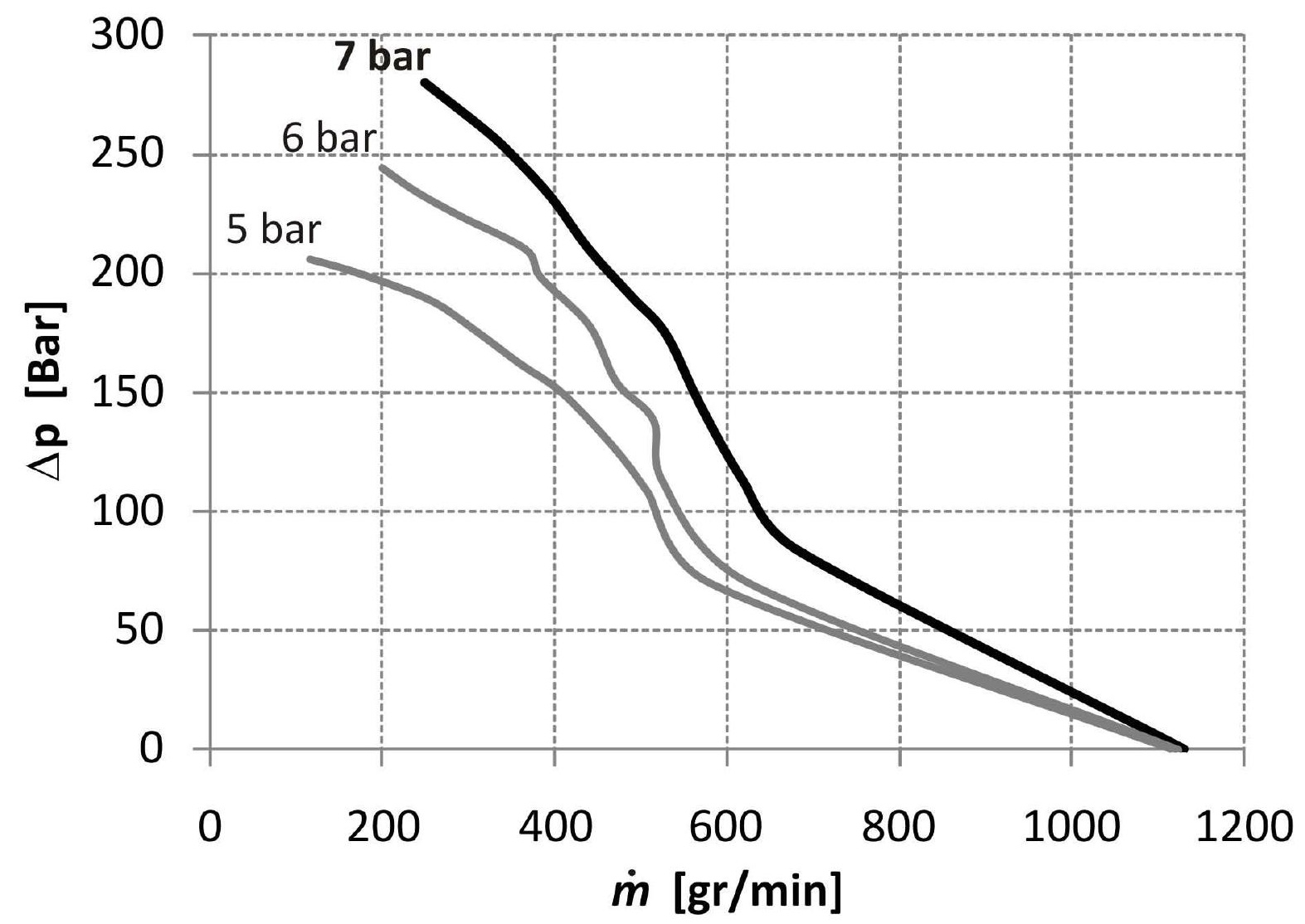



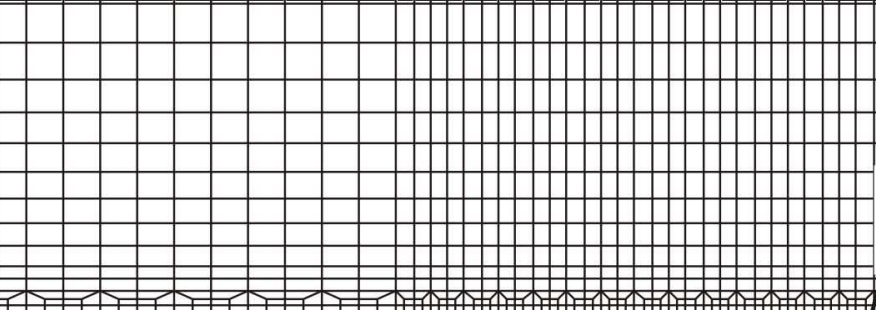

\section{interface (allows the relative motion between mesh zones)}

\section{ationary surface}

ows the creation of

$v$ mesh rows)
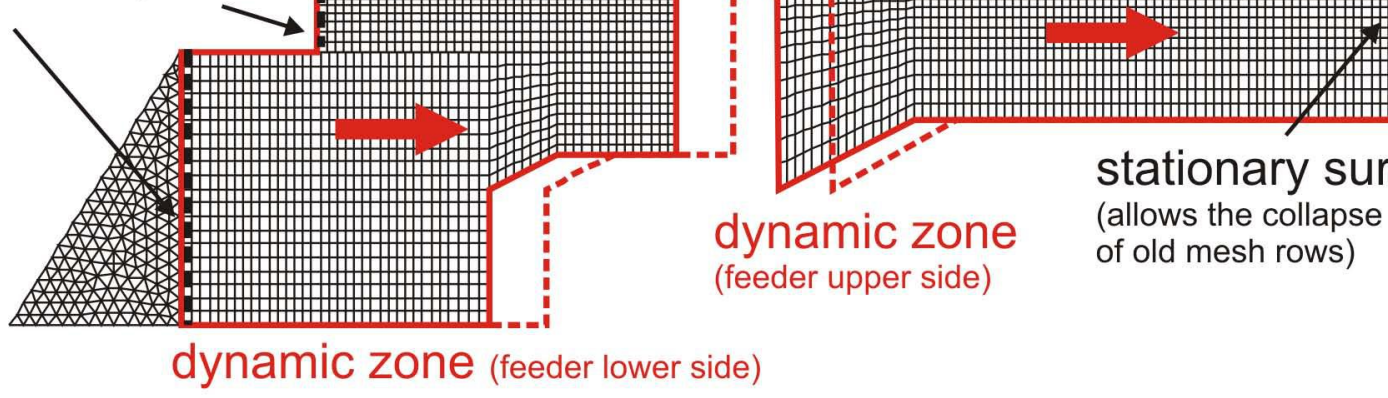

dynamic zone (feeder upper side)

(allows the collapse of old mesh rows)

dynamic zone (feeder lower side) 


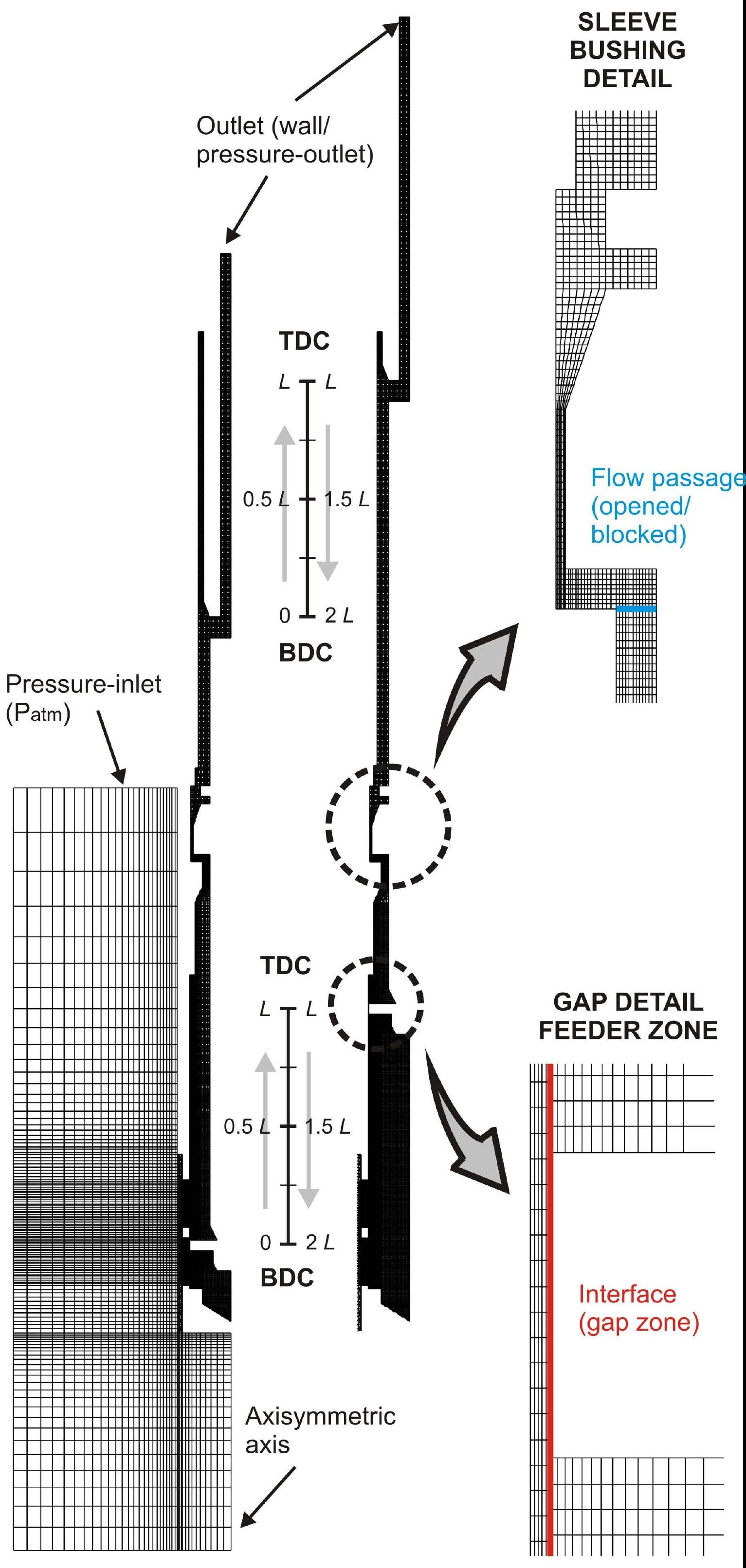




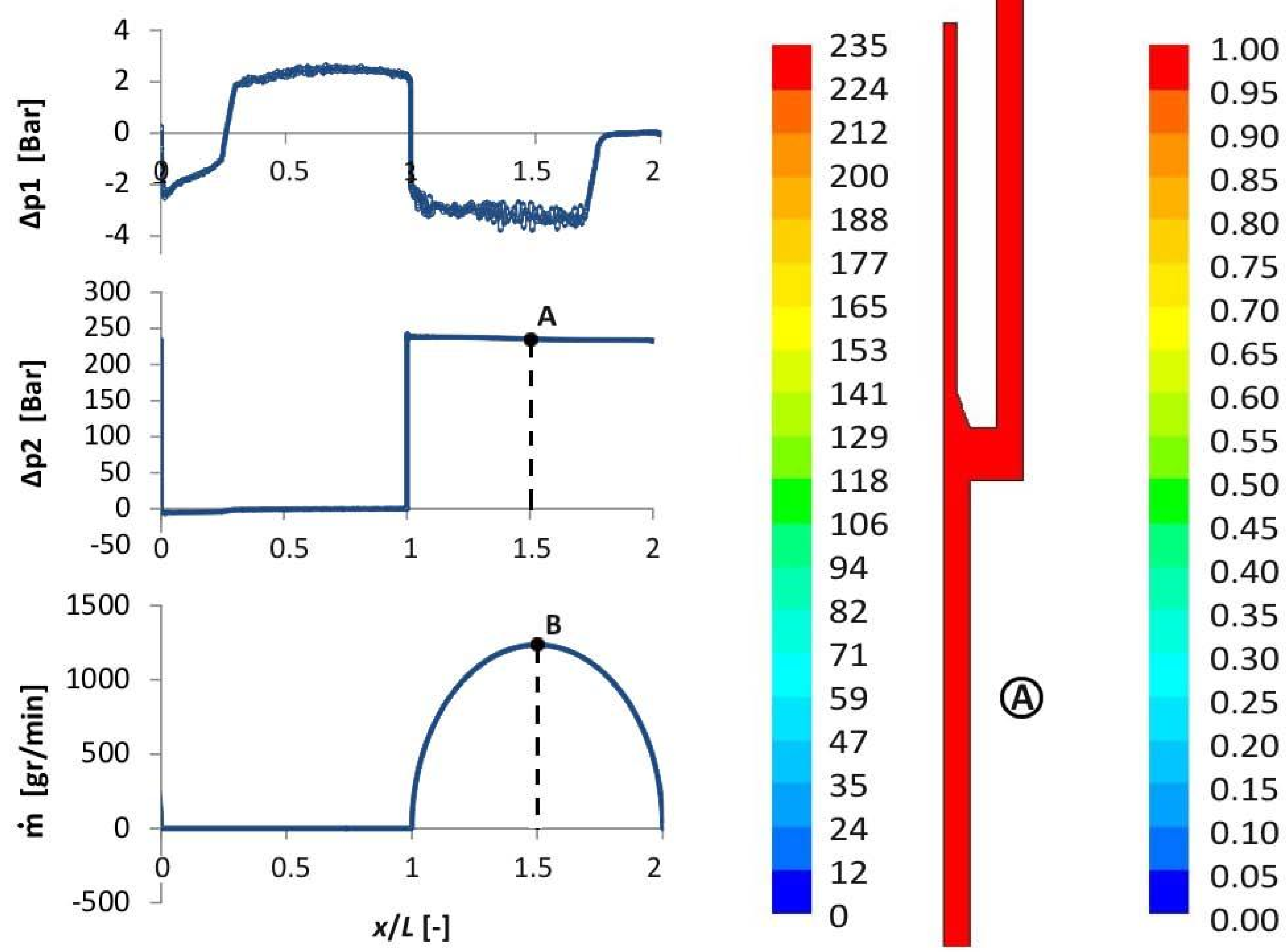




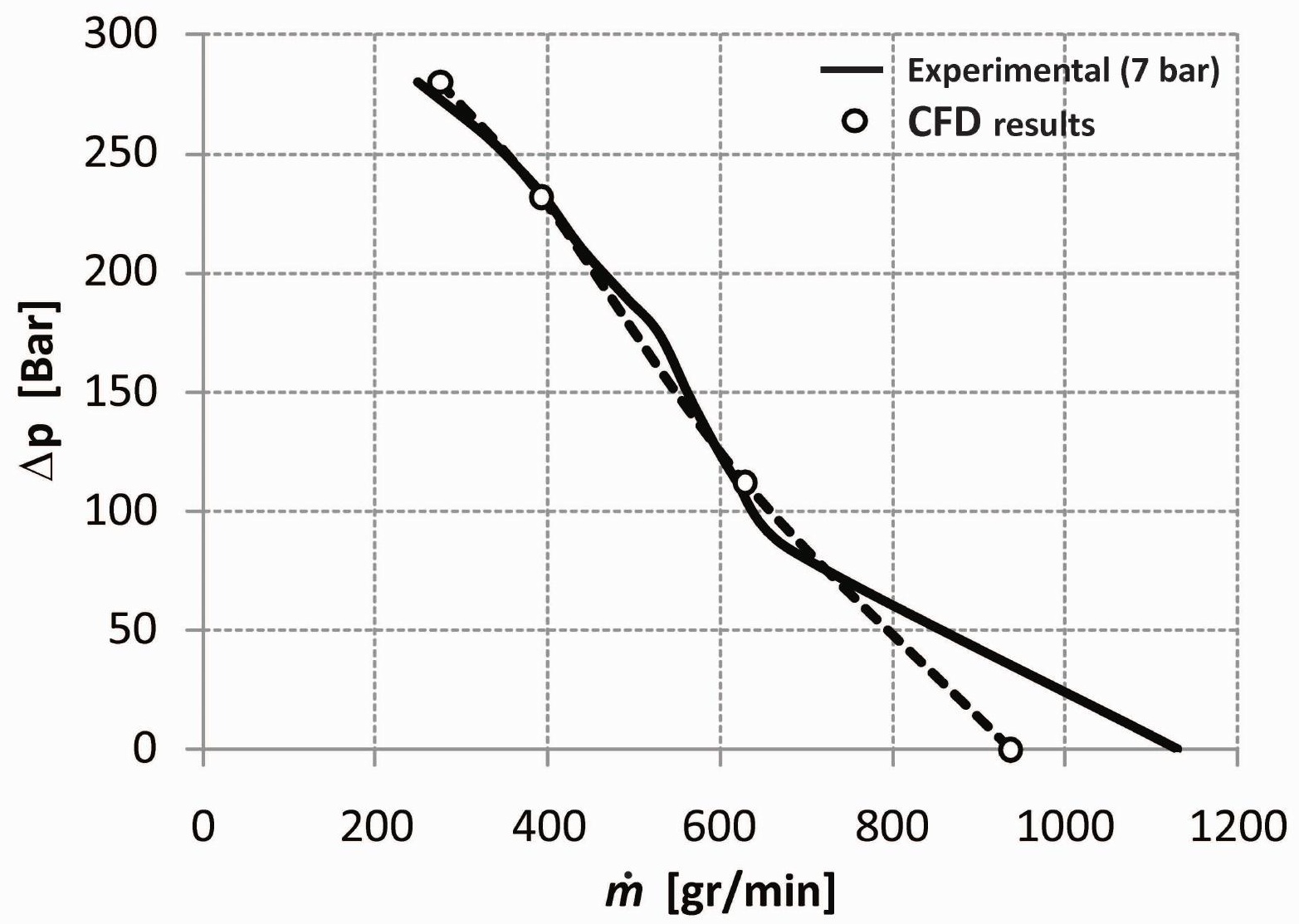




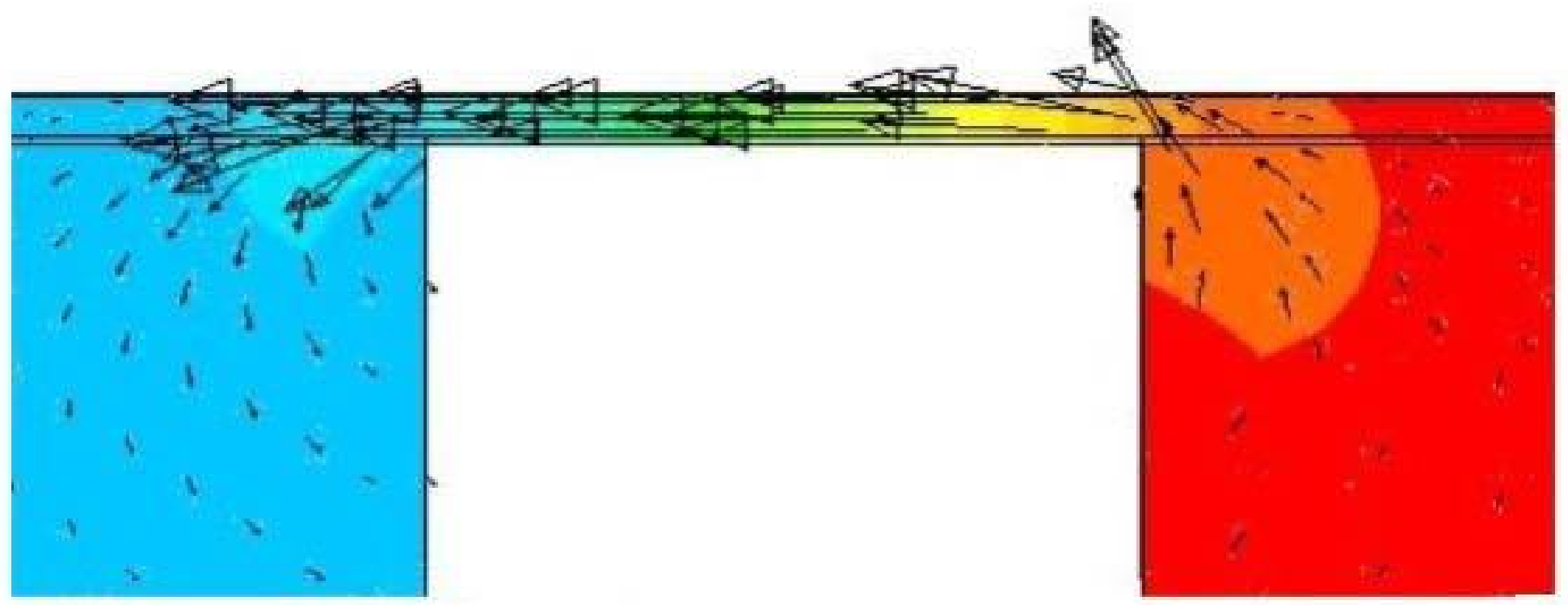

$\Delta \mathrm{p}$ [bar]

$\begin{array}{llllllll}-1.00 & -0.38 & 0.24 & 0.88 & 1.47 & 2.09 & 2.71 & 3.12\end{array}$ 


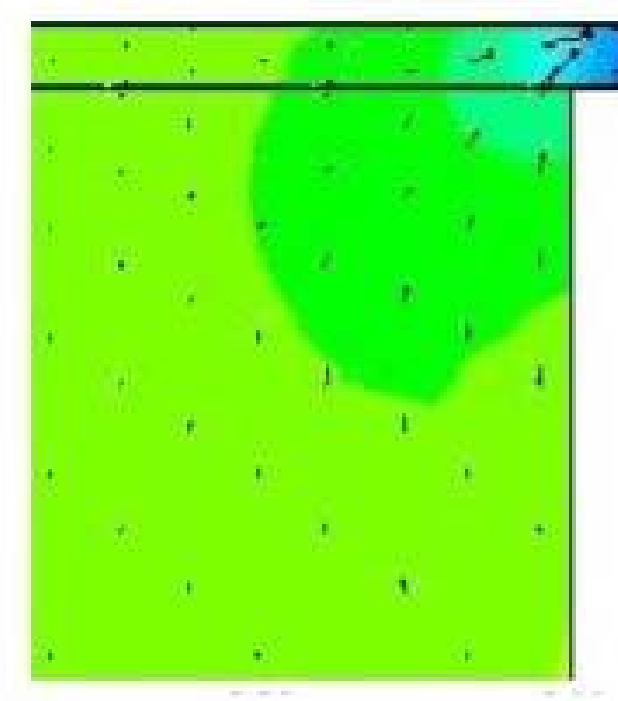

$\Delta \mathrm{p}$ [bar]

$\begin{array}{llllllll}1.00 & -0.68 & -0.32 & 0.01 & 0.36 & 0.89 & 1.02 & 1.26\end{array}$ 


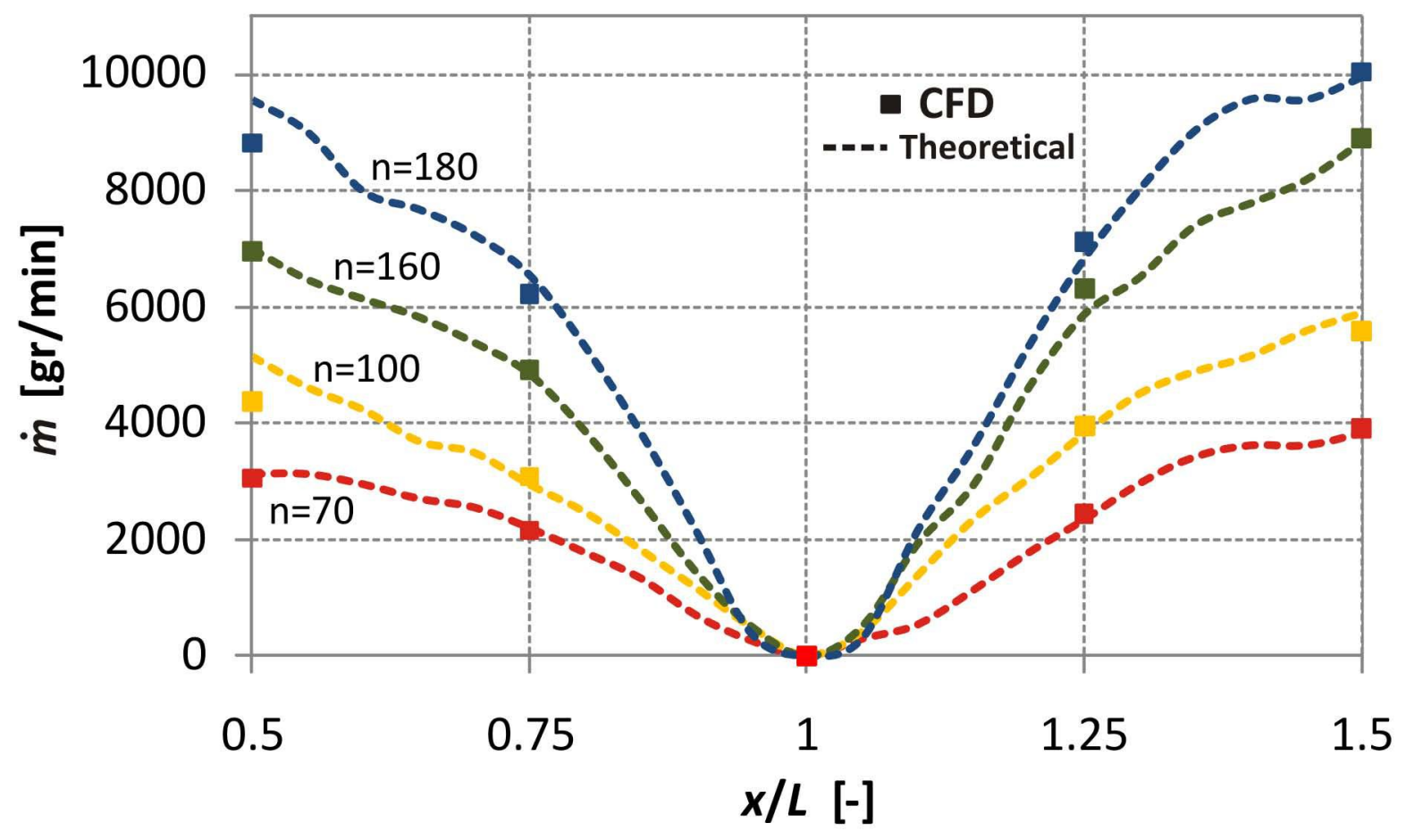




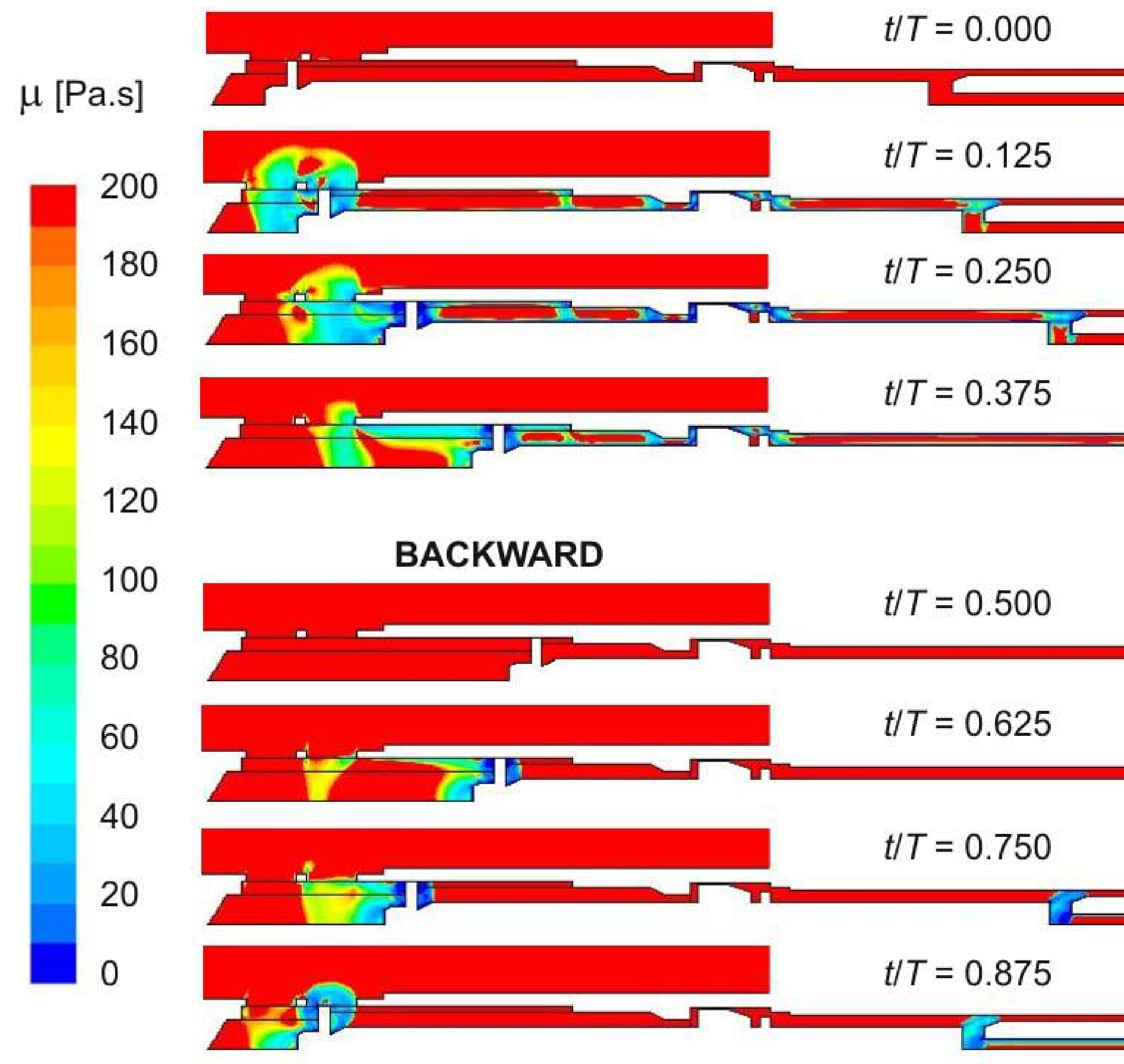


FORWARD
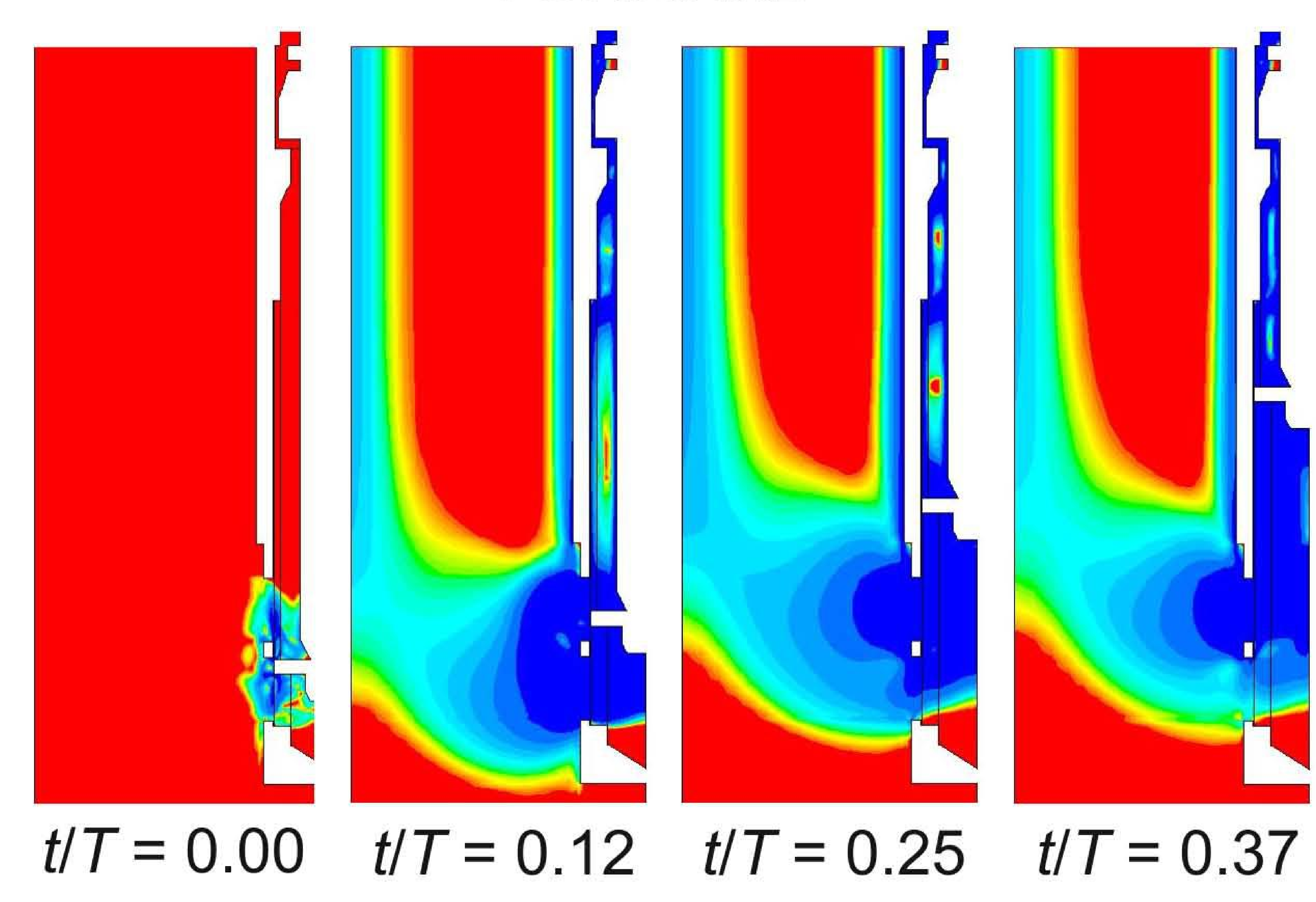

$t / T=0.37$

$\mu$ [Pa.s]
BACKWARD

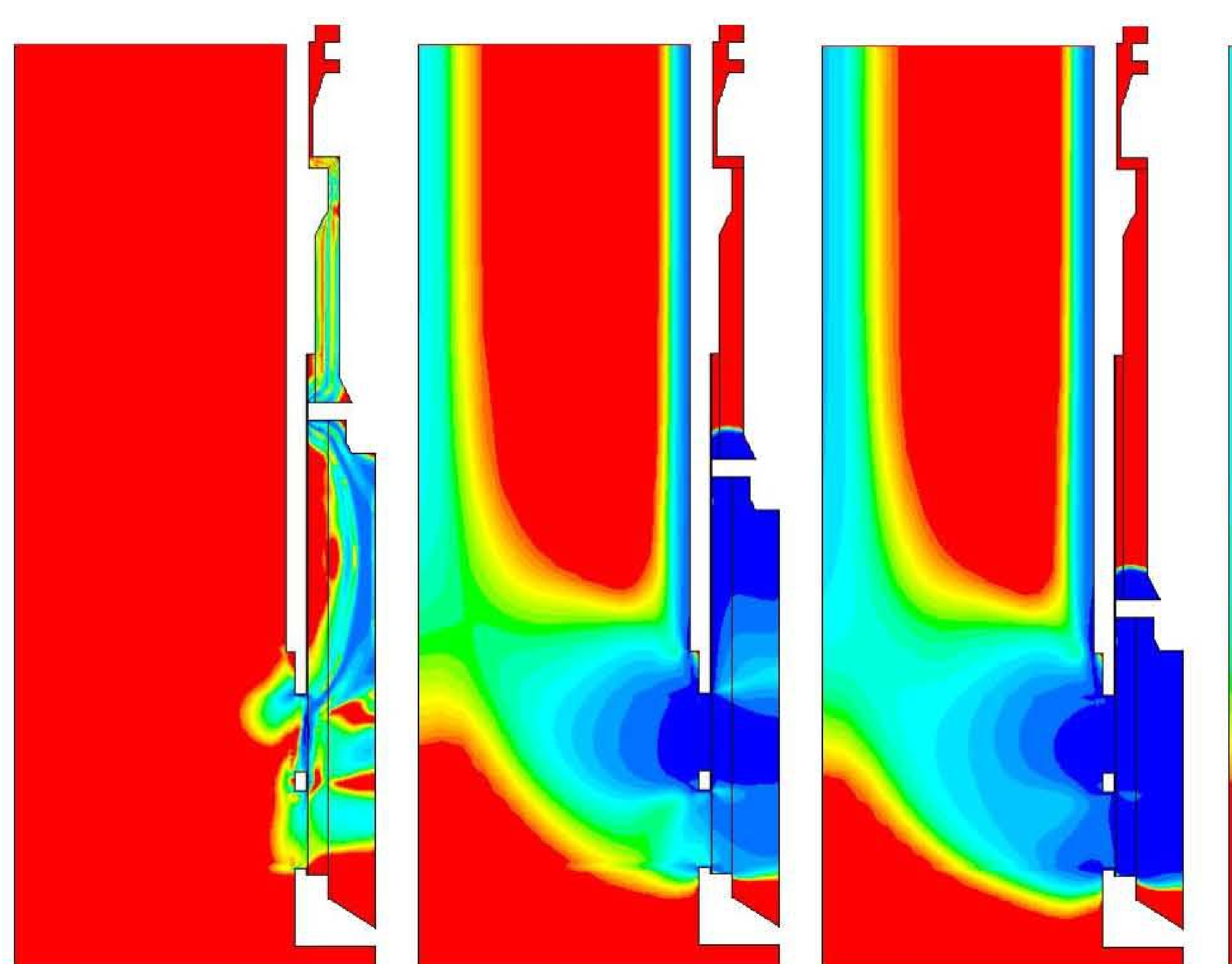

$t / T=0.50$

$t / T=0.62$

$t / T=0.75$

$t / T=0.87$ $\mu$ [Pa.s] 


\section{FORWARD $(x / L=0.5)$}
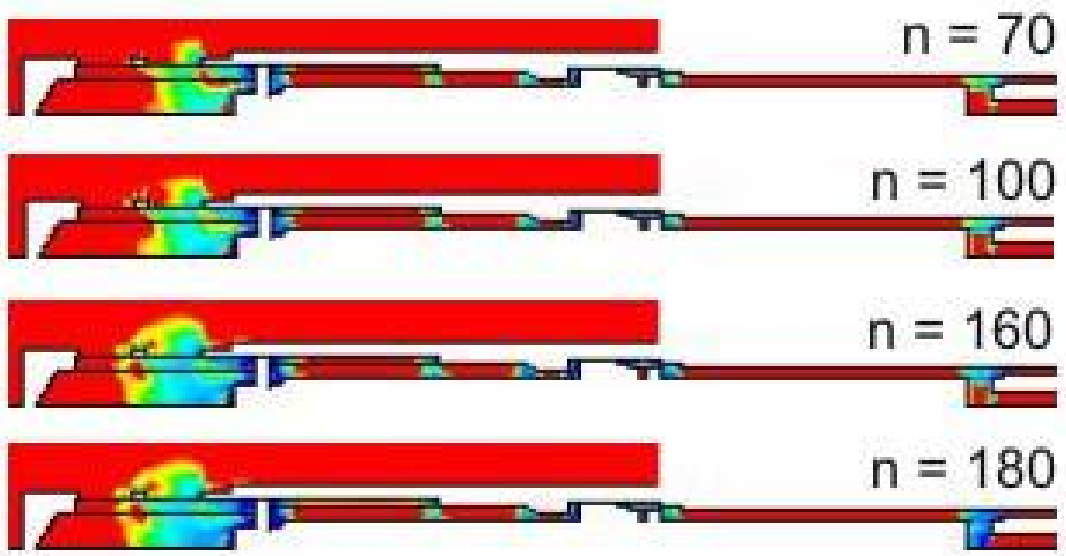

\section{BACKWARD $(x / L=1.5)$}
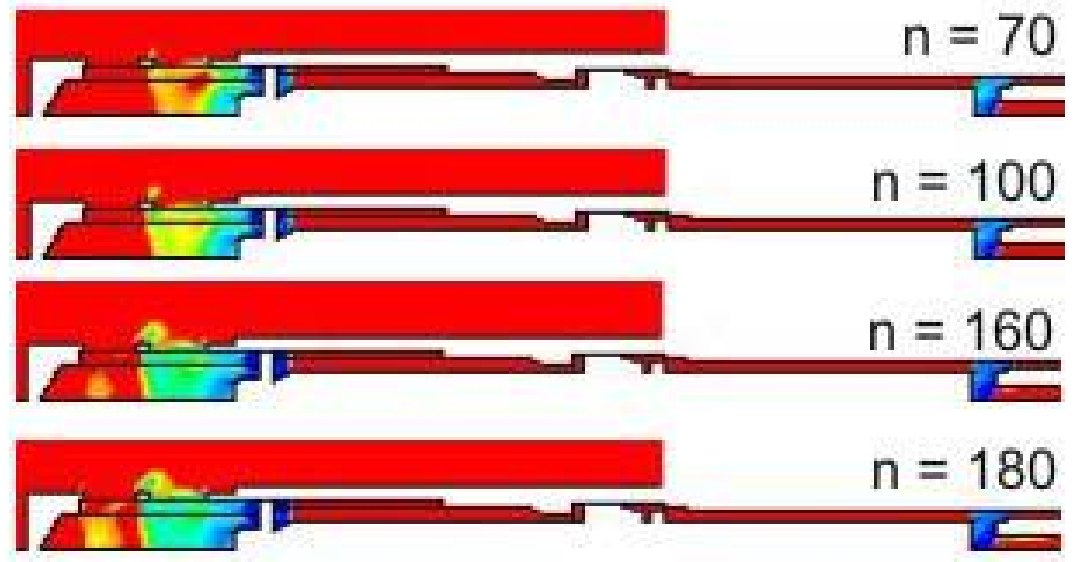

$\mu$ [Pa.s]

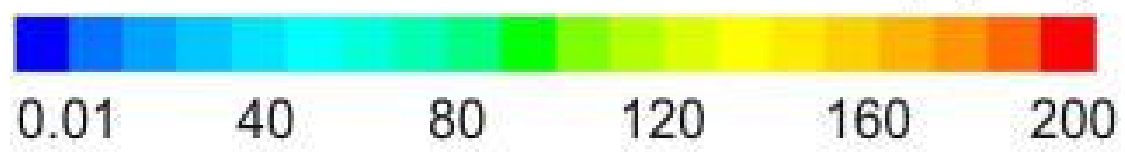


This document is a pre-print version of the scientific paper published by Elsevier. It has been released by the authors to fulfill all the publisher requirements established for Article Sharing: https://www.elsevier.com/about/policies/sharing

\section{(9) $\Theta \Theta \Theta$}

(C) 2019. This manuscript version is made available under the Creative Commons Attribution-NonCommercial-NoDerivatives 4.0 International License (CC-BY-NC-ND 4.0 license) http://creativecommons.org/licenses/by-nc-nd/4.0/ 